\title{
Probing Molecular Interactions in Functionalized Asymmetric Quaternary Ammonium Based Dicationic Ionic Liquids
}

\author{
Prakash L. Verma ${ }^{1}$, Libero J. Bartolotti ${ }^{2}$ and Shridhar P. Gejji ${ }^{1 *}$ \\ ${ }^{1}$ Department of Chemistry, Savitribai Phule Pune University, Pune 411 007, India \\ ${ }^{2}$ Department of Chemistry, East Carolina University, Greenville, North Carolina 27858-4353, USA
}

\section{Supporting Information}

Figure S1 Optimized [DRR1] (Tf2N) $)_{2}$ conformers (a), (b), (c), (d), (e), (f), (g) and (h) with relative stabilization energies (in $\mathrm{kJ} \mathrm{mol}^{-1}$ ) given in parentheses.

Figure S2 Optimized [DRR2](Tf2N) ${ }_{2}$ conformers (a), (b), (c), (d), (e), (f), (g) and (h) with relative stabilization energies (in $\mathrm{kJ} \mathrm{mol}^{-1}$ ) given in parentheses.

Figure S3 Optimized [DRR3](Tf2N) ${ }_{2}$ conformers (a), (b), (c), (d), (e), (f), (g) and (h) with relative stabilization energies (in $\mathrm{kJ} \mathrm{mol}^{-1}$ ) given in parentheses.

Figure S4 Optimized [DRR4](Tf2N) ${ }_{2}$ conformers (a), (b), (c), (d), (e), (f), (g) and (h) with relative stabilization energies (in $\mathrm{kJ} \mathrm{mol}^{-1}$ ) given in parentheses.

Table S1(a) Hydrogen bonding distances (in Å) (prime and without prime referring to two anions) in dication- $\left(\mathrm{Tf}_{2} \mathrm{~N}\right)_{2}$ complexes.

Table S1(b) Selected bond- distances (in $\AA$ ) and -angles (in ${ }^{\circ}$ ) of isolated dications and dication$\left(\mathrm{Tf}_{2} \mathrm{~N}\right)_{2}$ complexes.

Table S1(c) Selected bond -distances and -angles in the isolated $\left[\mathrm{Tf}_{2} \mathrm{~N}\right]^{-}$anion and dication$\left(\mathrm{Tf}_{2} \mathrm{~N}\right)_{2}$ complexes.

Figure S5 Calculated IR frequencies for dication, [DRR2 $]^{2+}$, anion [Tf $\mathrm{T}_{2} \mathrm{~N}^{-}$and [DRR2](Tf2N) ${ }_{2}$ complex in regions (a) $3400-3000 \mathrm{~cm}^{-1}$, and (b) $1600-800 \mathrm{~cm}^{-1}$.

Figure S6 Calculated IR frequencies for dication, [DRR3] ${ }^{2+}$, anion [Tf $\mathrm{T}_{2} \mathrm{~N}^{-}$and [DRR3](Tf2N) ${ }_{2}$ complex in regions (a) $3400-3000 \mathrm{~cm}^{-1}$, and (b) $1600-800 \mathrm{~cm}^{-1}$.

Figure S7 Calculated IR frequencies for dication, [DRR4] ${ }^{2+}$, anion [Tf $\mathrm{T}_{2} \mathrm{~N}^{-}$and [DRR4](Tf2N) 2 complex in regions (a) $3400-3000 \mathrm{~cm}^{-1}$, and (b) $1600-800 \mathrm{~cm}^{-1}$.

Table S2 NBO charges (in au) in the isolated $\mathrm{Tf}_{2} \mathrm{~N}^{-}$anion and dication- $\left(\mathrm{Tf}_{2} \mathrm{~N}\right)_{2}$ complexes.

Figure S8 Difference MED maps in the [DRR2] $\left(\mathrm{Tf}_{2} \mathrm{~N}\right)_{2}$ complex in the plane passing through interacting $\mathrm{C}-\mathrm{H}$ and $\mathrm{S}-\mathrm{O}$ bonds. Contours in the range of \pm 0.001 to \pm 0.009 a.u. are shown along with the BCPs in the MED topography. 
Figure S9 Difference MED maps in the [DRR3] $\left(\mathrm{Tf}_{2} \mathrm{~N}\right)_{2}$ complex in the plane passing through interacting $\mathrm{C}-\mathrm{H}$ and $\mathrm{S}-\mathrm{O}$ bonds. Contours in the range of \pm 0.001 to \pm 0.009 a.u. are shown along with the BCPs in the MED topography.

Figure S10 Difference MED maps in the [DRR4] $\left(\mathrm{Tf}_{2} \mathrm{~N}\right)_{2}$ complex in the plane passing through interacting $\mathrm{C}-\mathrm{H}$ and $\mathrm{S}-\mathrm{O}$ bonds. Contours in the range of \pm 0.001 to \pm 0.009 a.u. are shown along with the BCPs in the MED topography.

Figure S11 Schematic representation the topological graphics of the critical points (a) and of the electrostatic potential map, Isovalue 0.0004 a.u. (b) for the [DRR1] $\left(\mathrm{Tf}_{2} \mathrm{~N}\right)_{2}$ complexes. The medium gray, blue, red, and white spheres represent the carbon, nitrogen, oxygen, and hydrogen atoms, respectively.

Figure S12 Schematic representation the topological graphics of the critical points (a) and of the electrostatic potential map, Isovalue 0.0004 a.u. (b) for the [DRR2] $\left(\mathrm{Tf}_{2} \mathrm{~N}\right)_{2}$ complexes. The medium gray, blue, red, and white spheres represent the carbon, nitrogen, oxygen, and hydrogen atoms, respectively.

Figure S13 Schematic representation the topological graphics of the critical points (a) and of the electrostatic potential map, Isovalue 0.0004 a.u. (b) for the [DRR3] $\left(\mathrm{Tf}_{2} \mathrm{~N}\right)_{2}$ complexes. The medium gray, blue, red, and white spheres represent the carbon, nitrogen, oxygen, and hydrogen atoms, respectively.

Figure S14 Schematic representation the topological graphics of the critical points (a) and of the electrostatic potential map, Isovalue $0.0004 \mathrm{au}$ (b) for the [DRR4] $\left(\mathrm{Tf}_{2} \mathrm{~N}\right)_{2}$ complexes. The medium gray, blue, red, and white spheres represent the carbon, nitrogen, oxygen, and hydrogen atoms, respectively.

Figure S15 Dispersive, polarization, steric energy contributions to binding energies accompanying the DIL.

Figure S16 A plot of glass transition temperature $\left(\mathrm{T}_{\mathrm{g}}\right)$ as a function of binding energies accompanying the DIL.

Figure S17 Viscosity, $\eta$ (mPa.s) plotted against the change in enthalpy, $\Delta \mathrm{H}$ (in kcal mol${ }^{-1}$ ). 


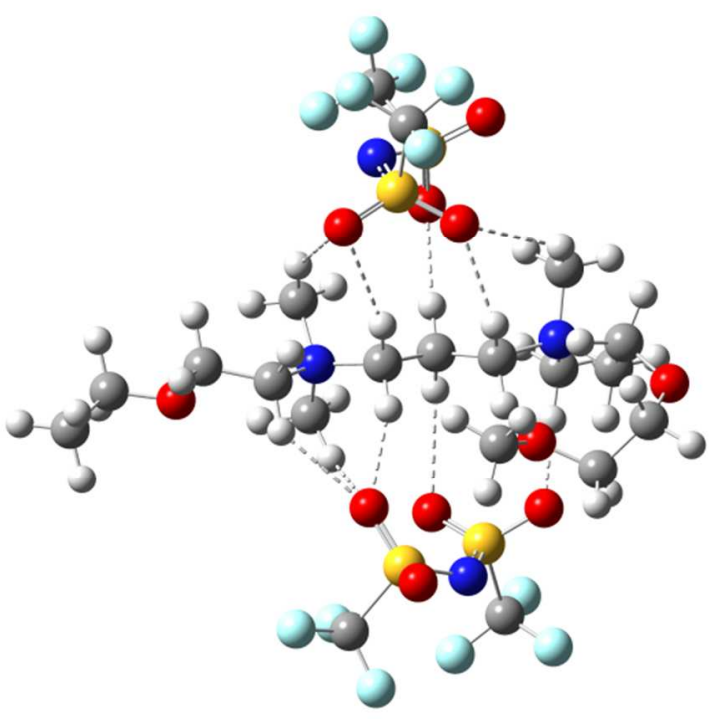

(a) 0.0

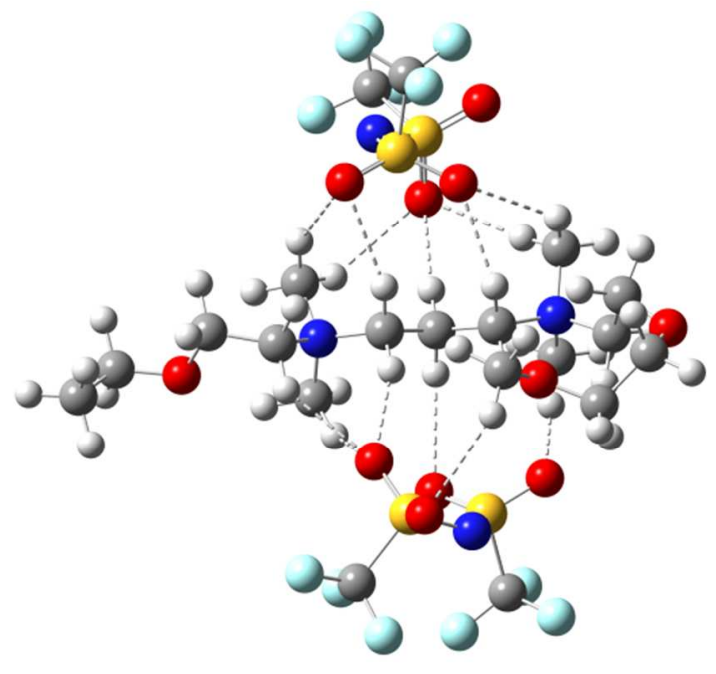

(c) 6.1

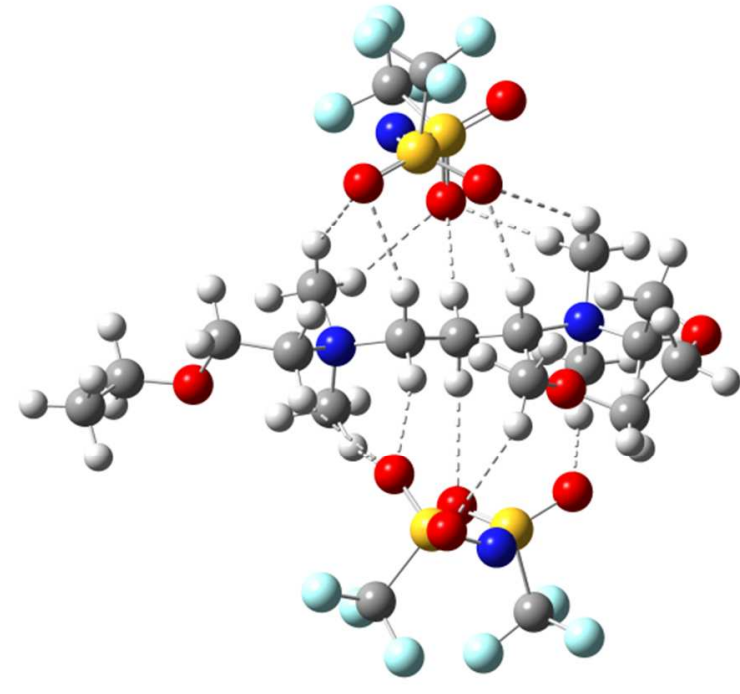

(b) 2.8

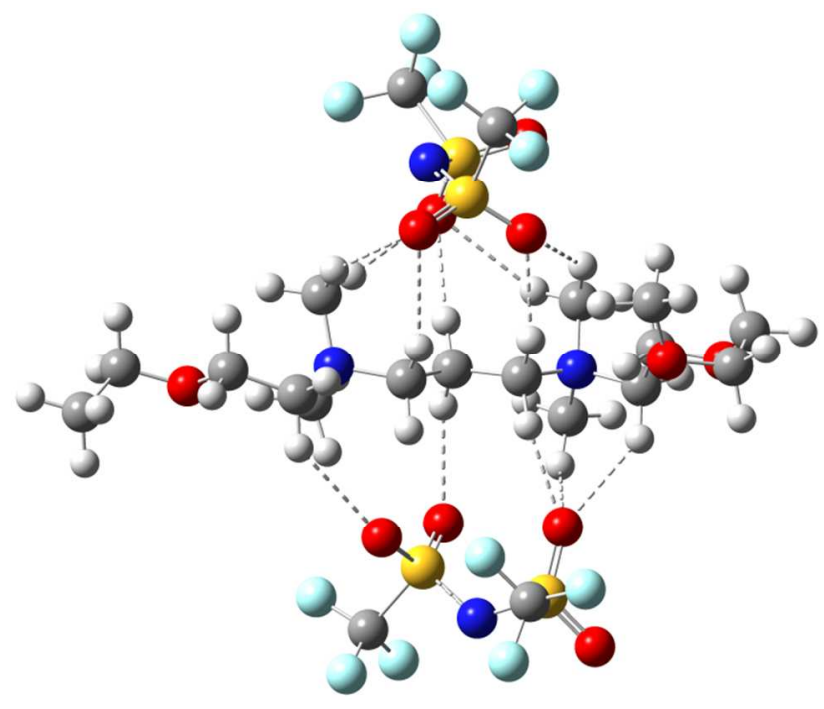

(d) 6.1 


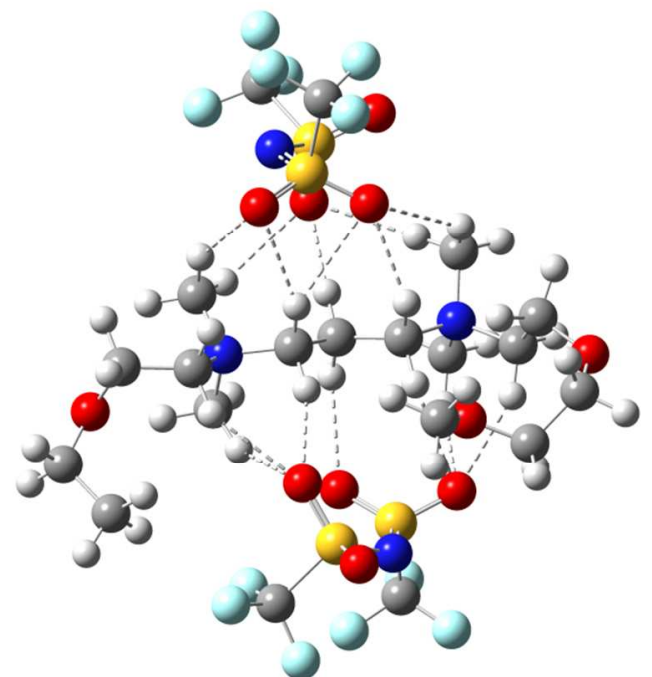

(e) 7.0

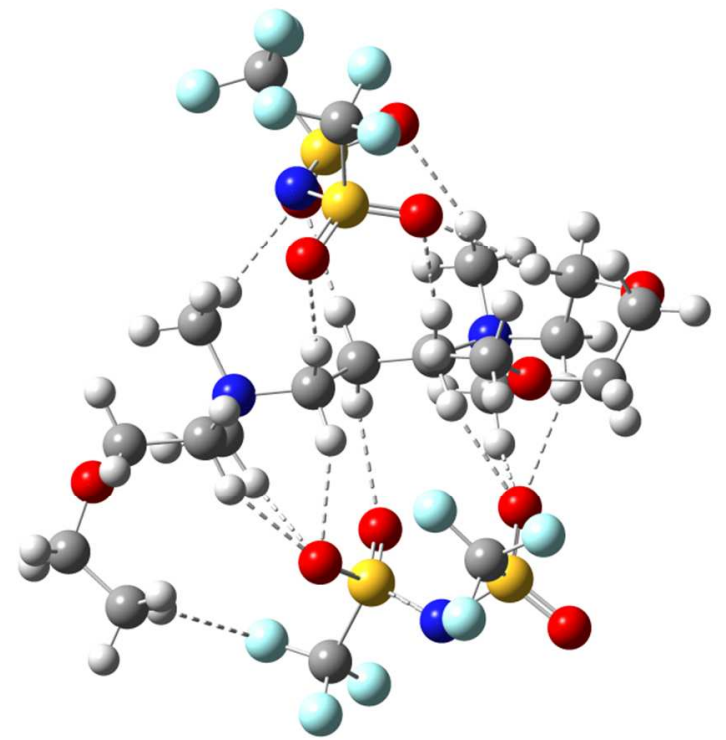

(g)11.6

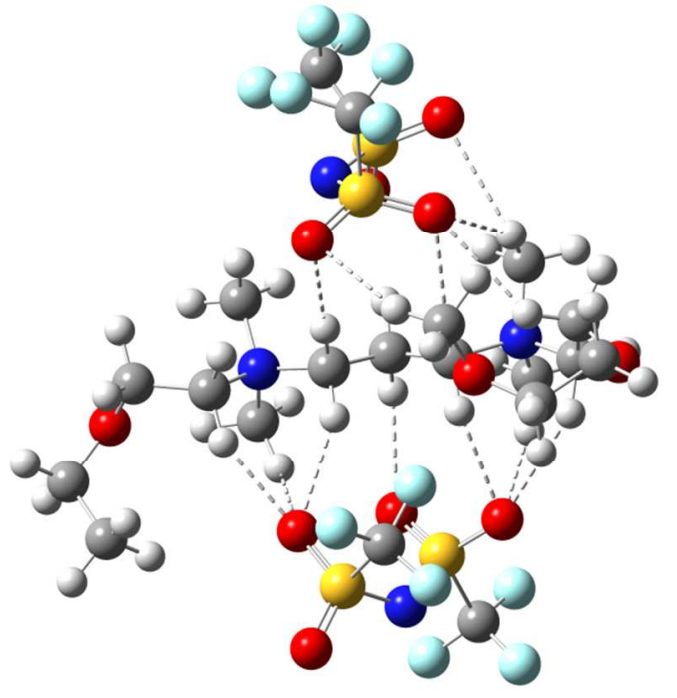

(f) 7.8

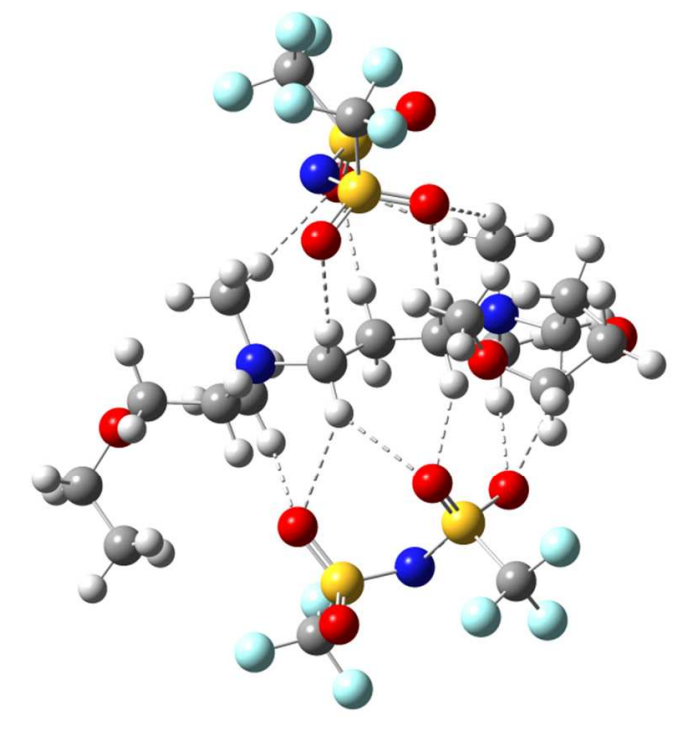

(h) 17.2 


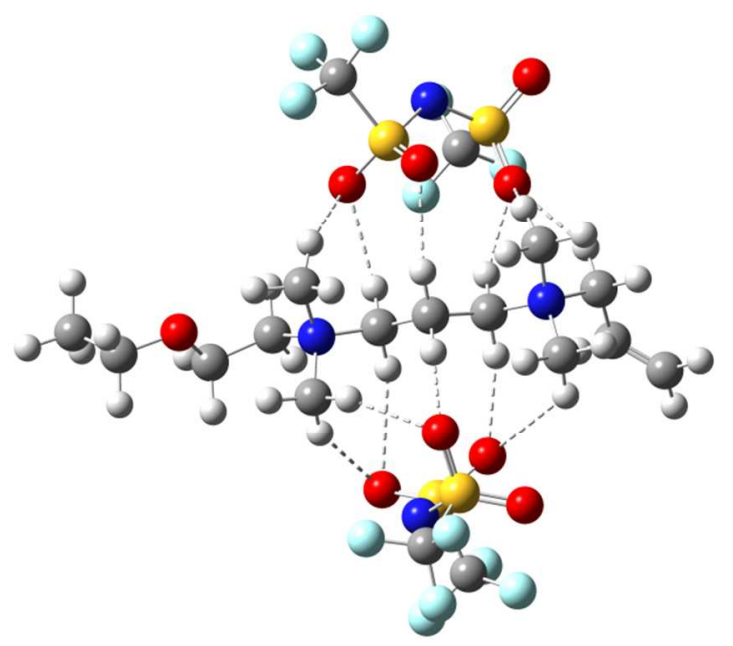

(a) 0.0

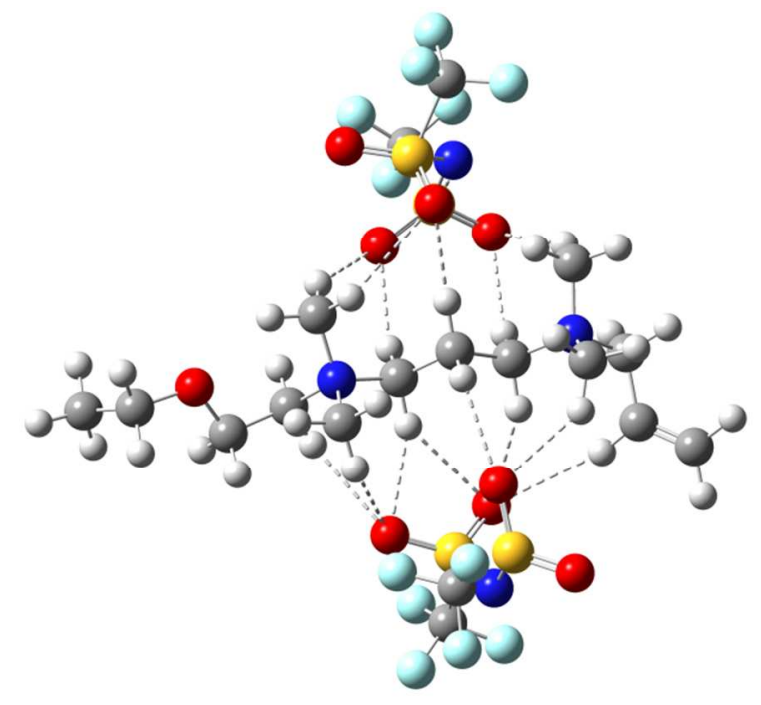

(c) 0.7

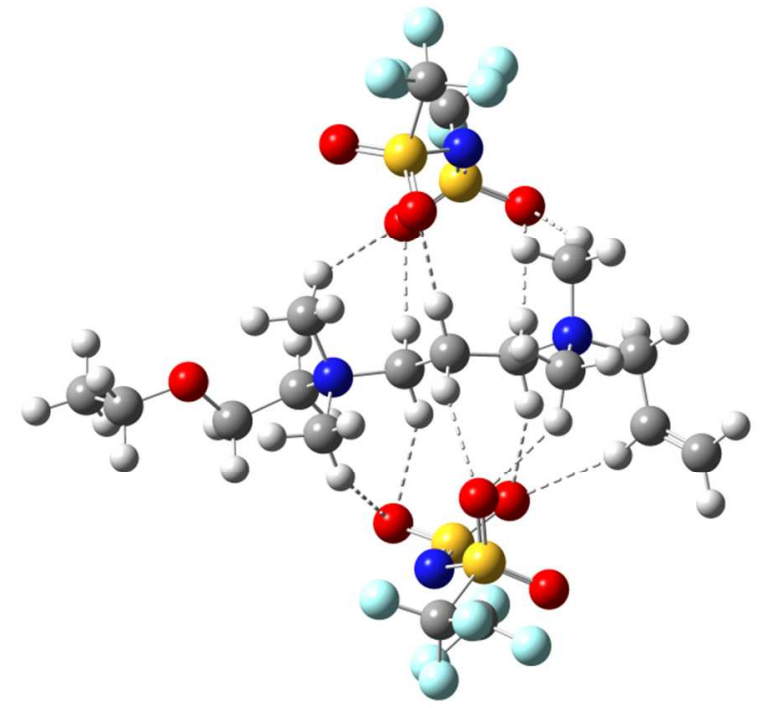

(b) 0.7

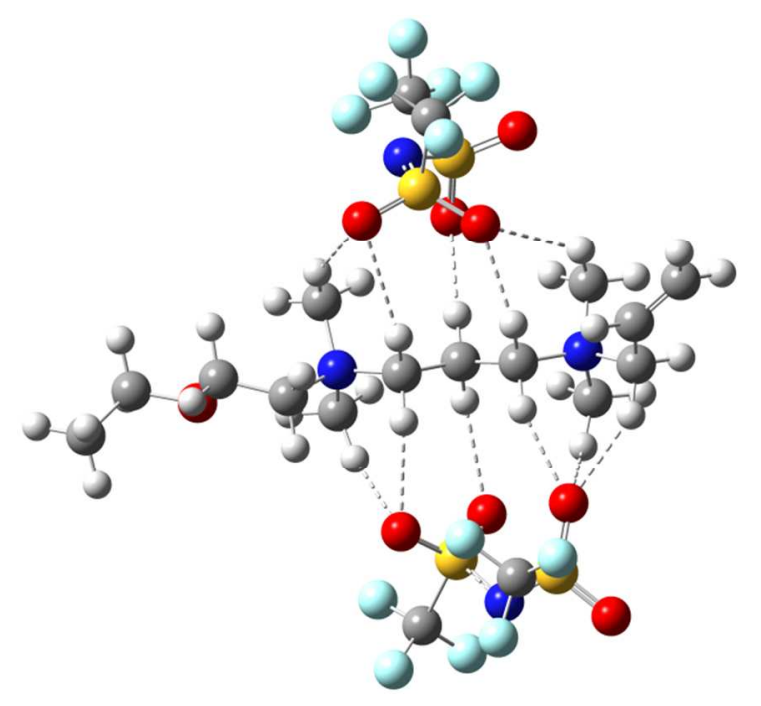

(d) 1.4 


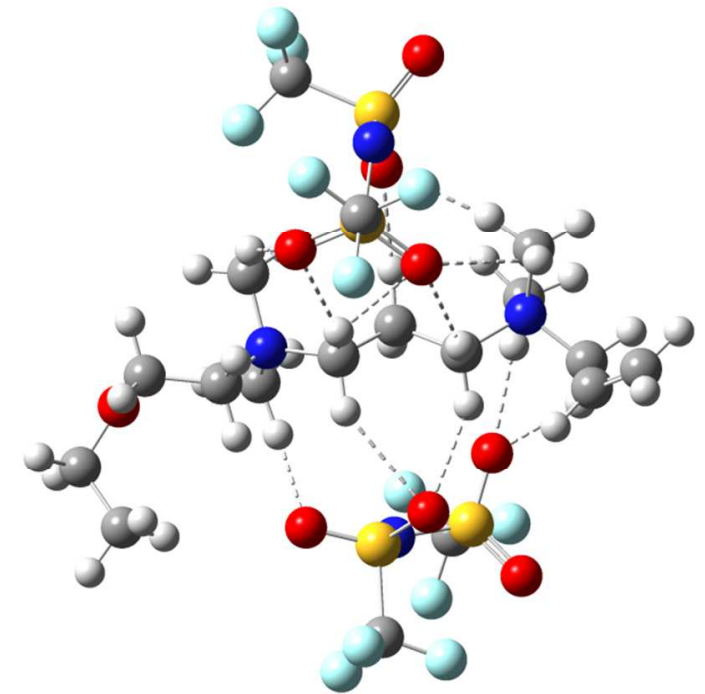

(e) 6.6

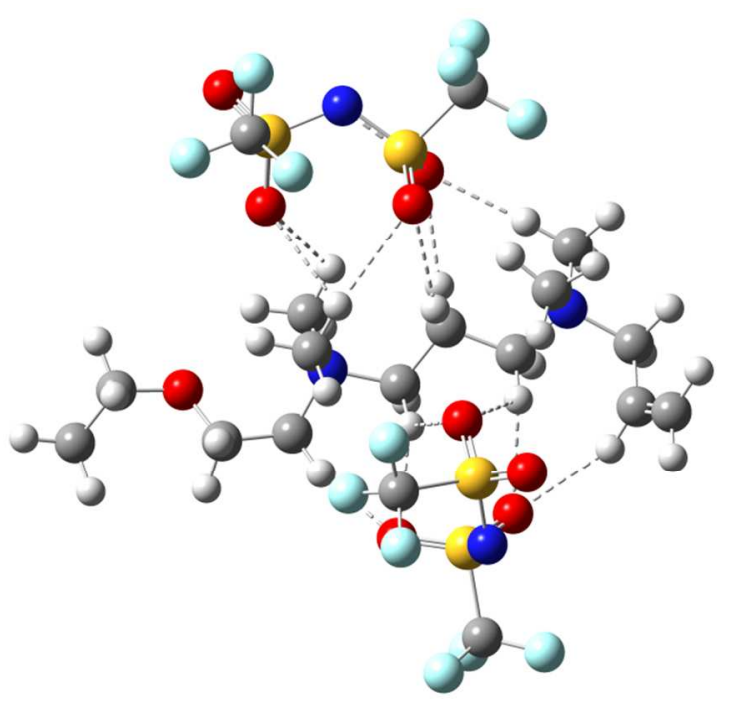

(g) 9.7

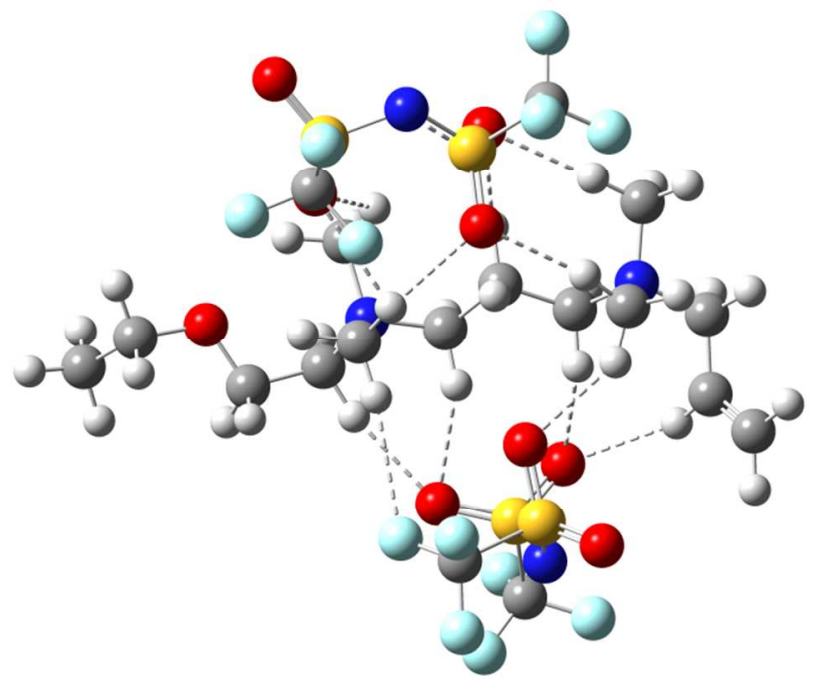

(f) 6.6

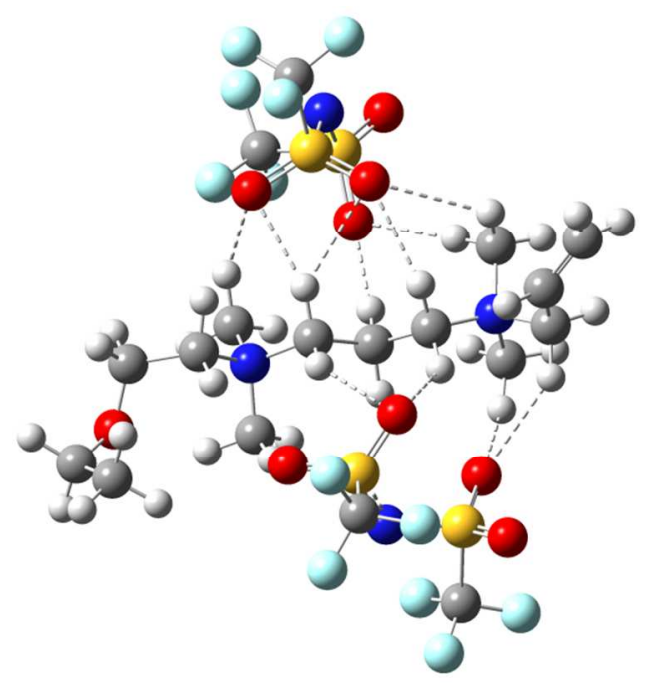

(h) 23.6 


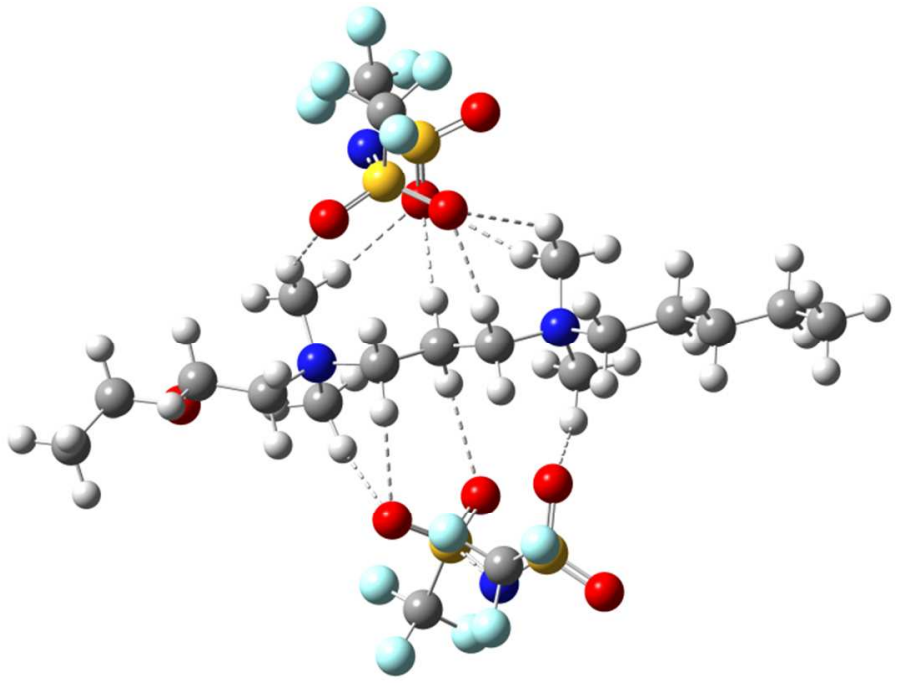

(a) 0.0

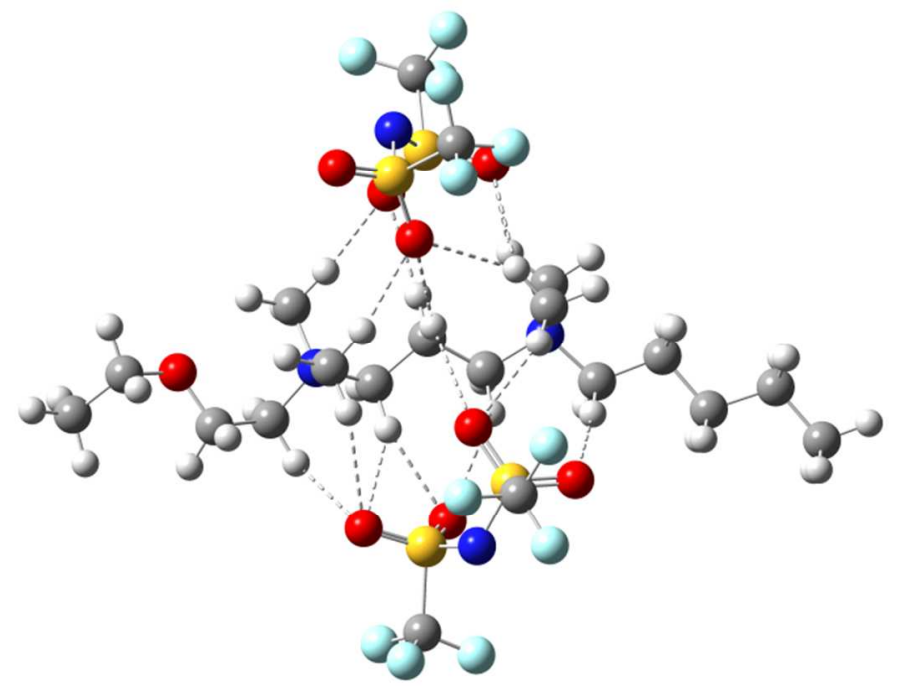

(c) 12.0

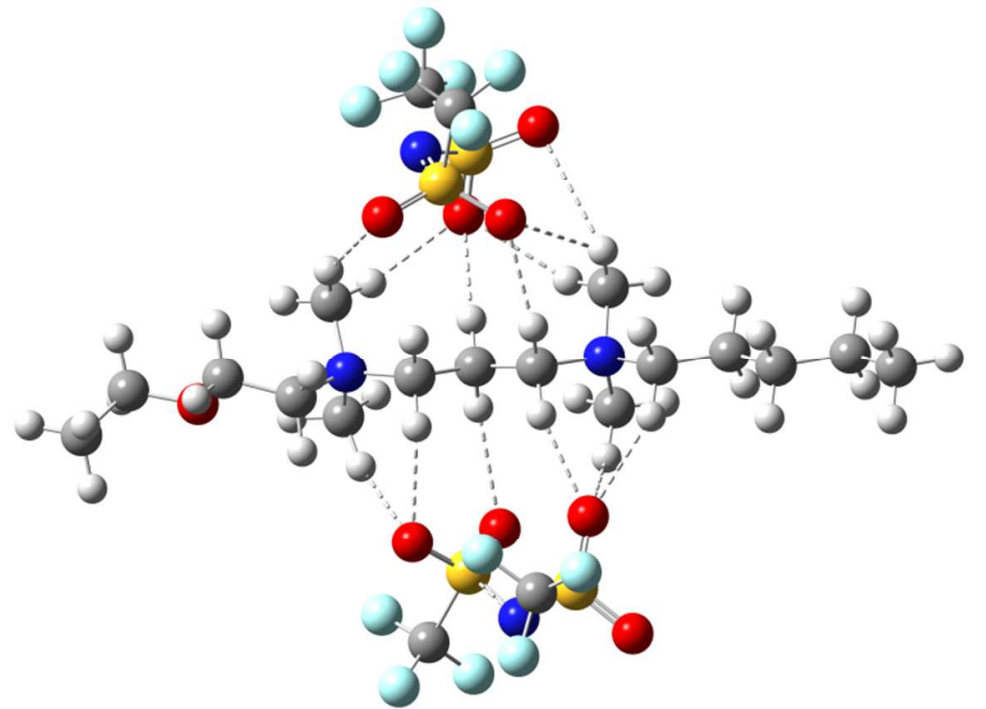

(b) 9.0

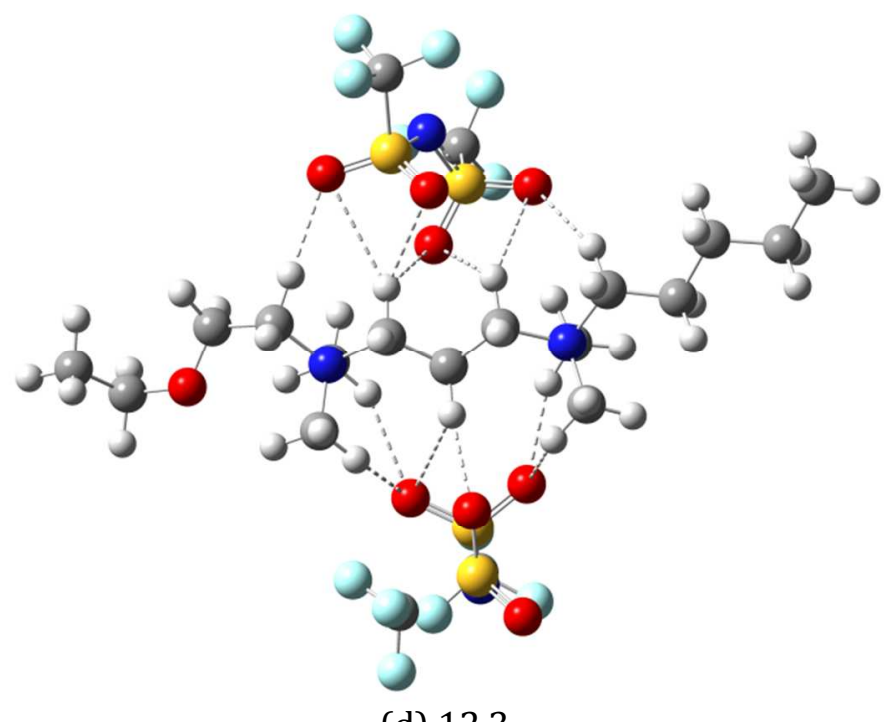

(d) 12.3 


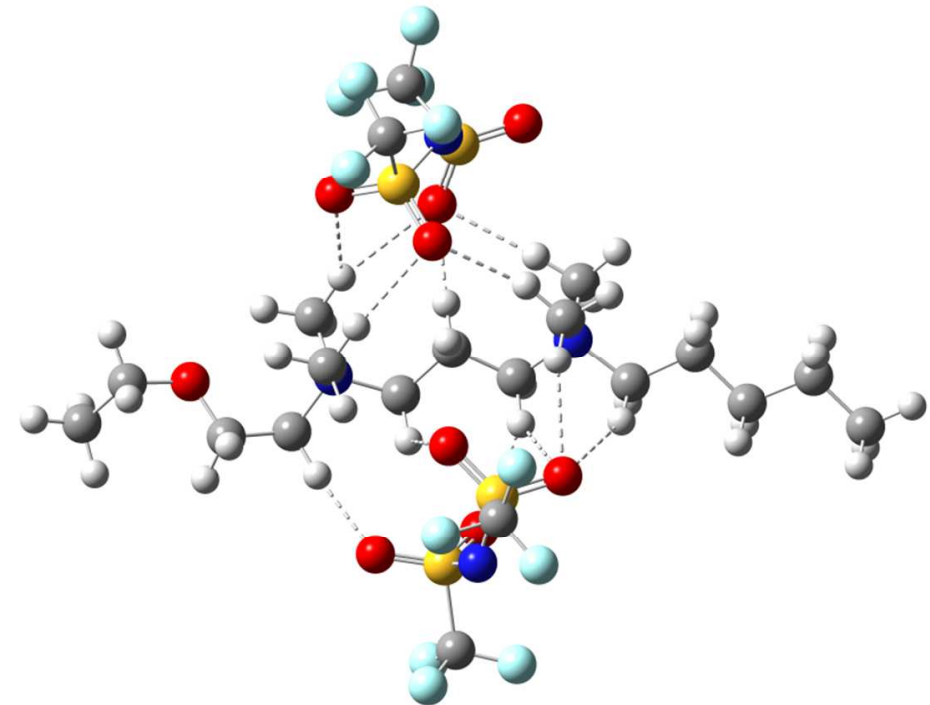

(e) 10.0

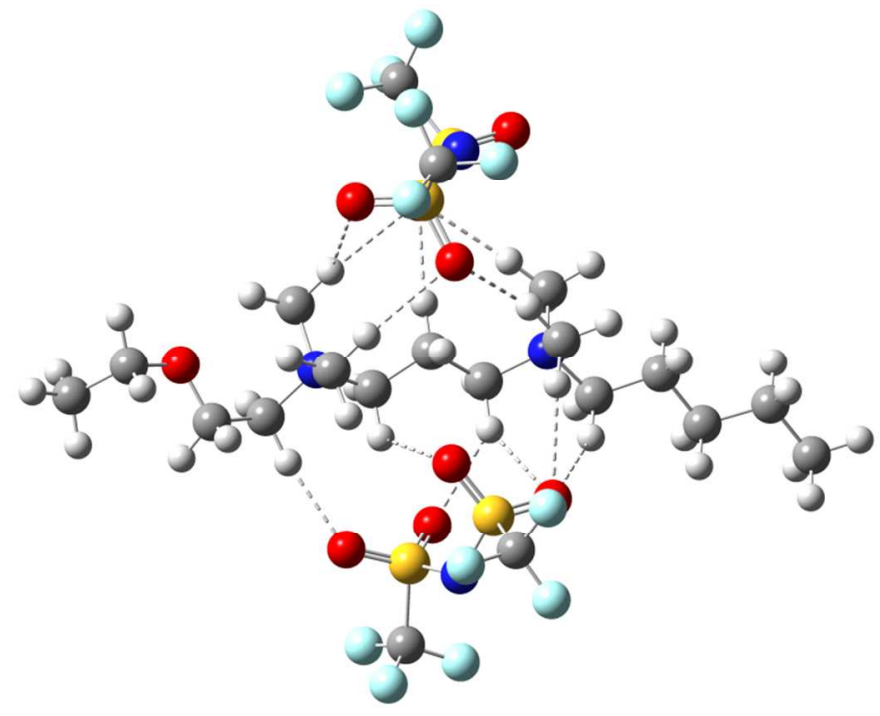

(g) 11.0

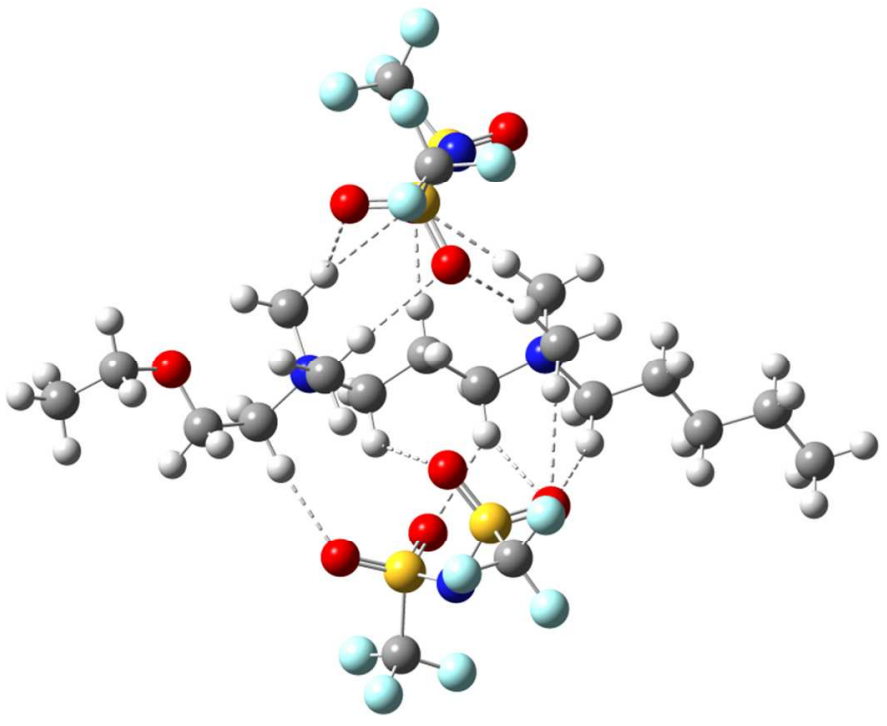

(f) 10.5

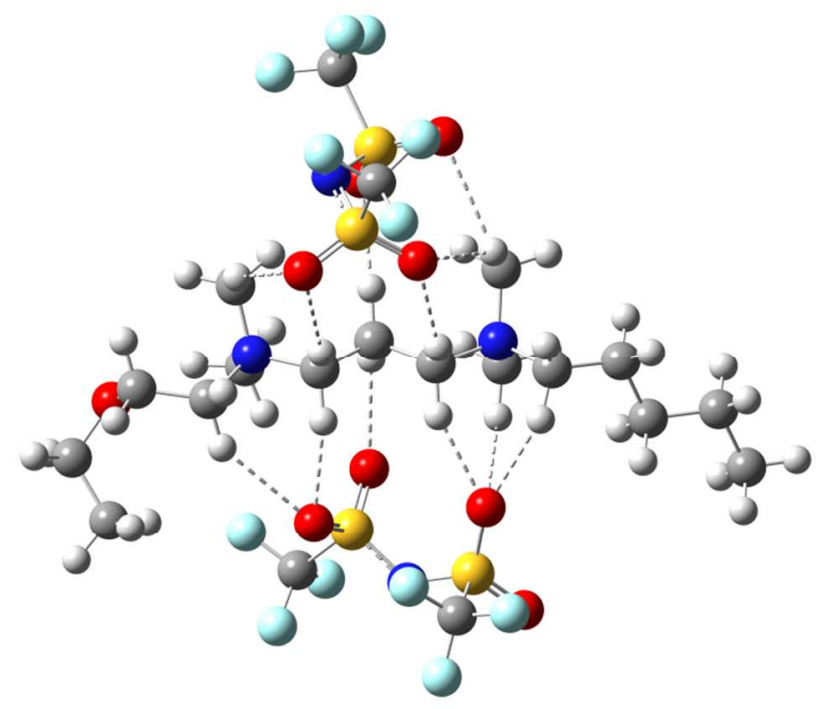

(h)11.4 


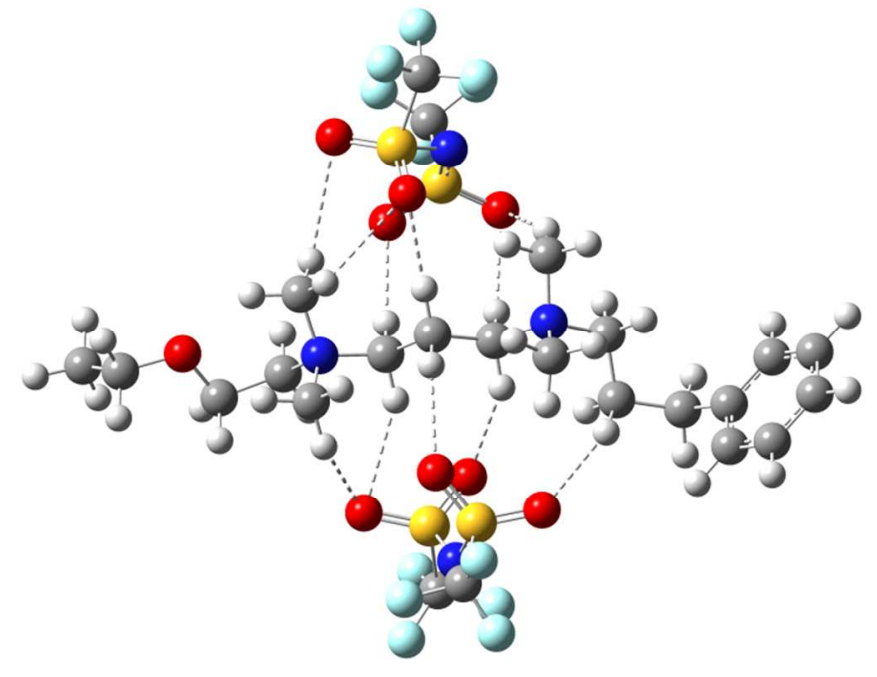

(a) 0.0

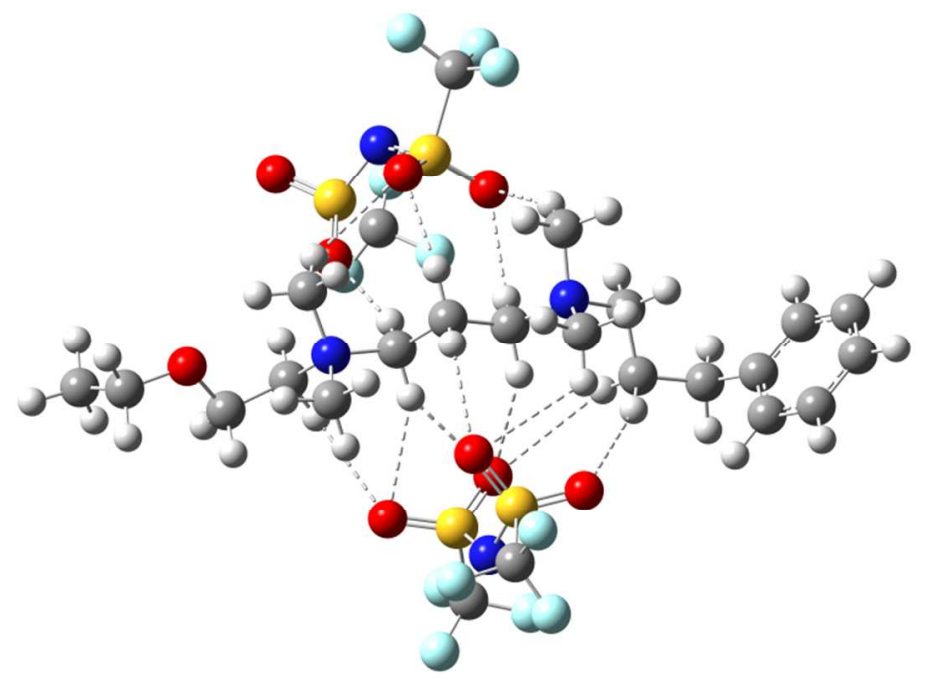

(c) 5.0

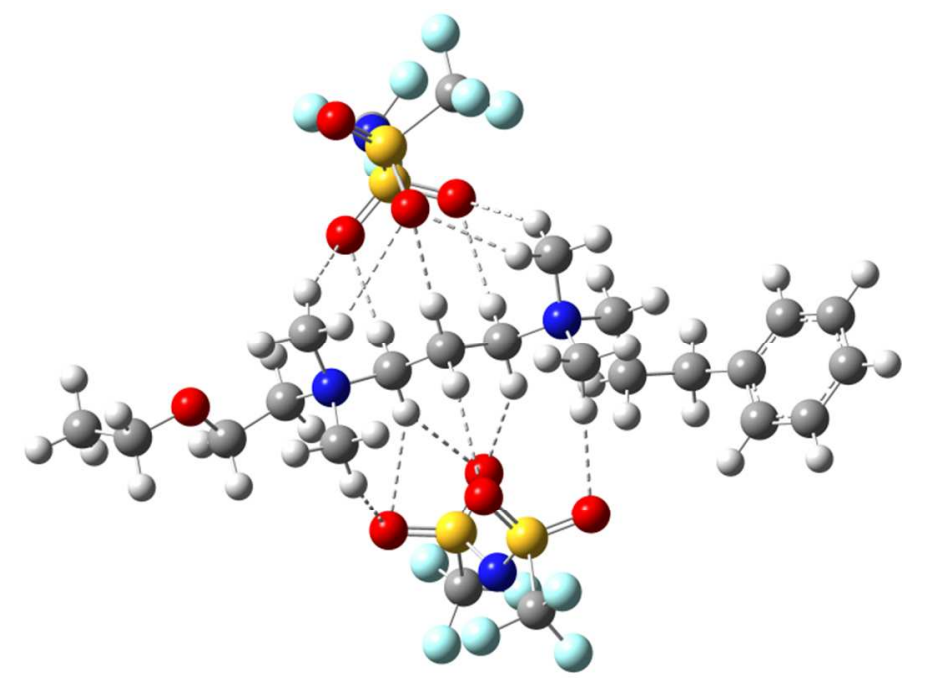

(b) 1.6

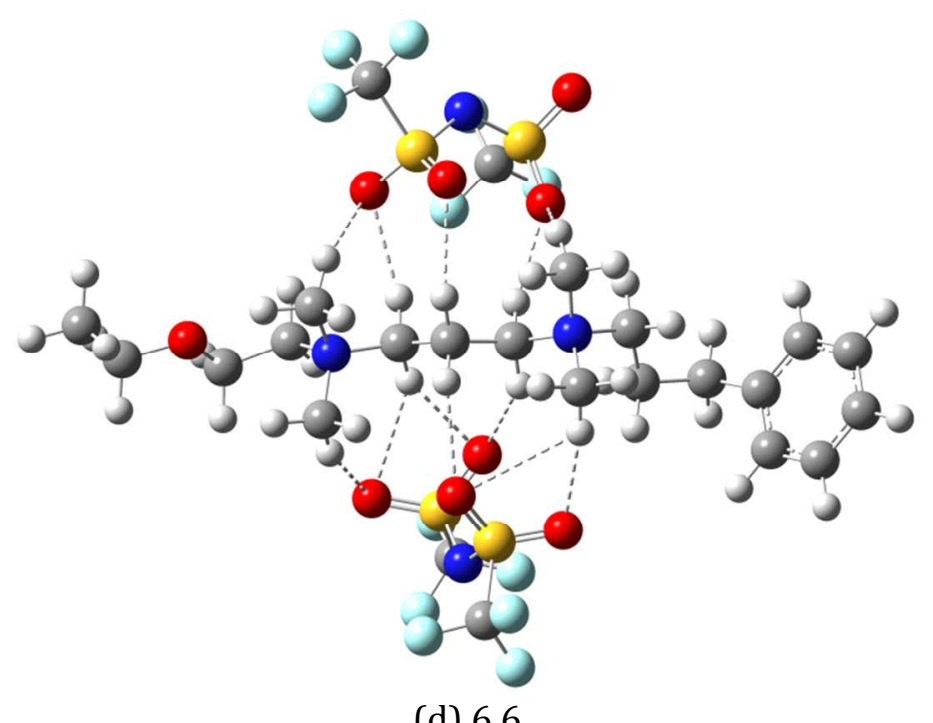

Continued... 


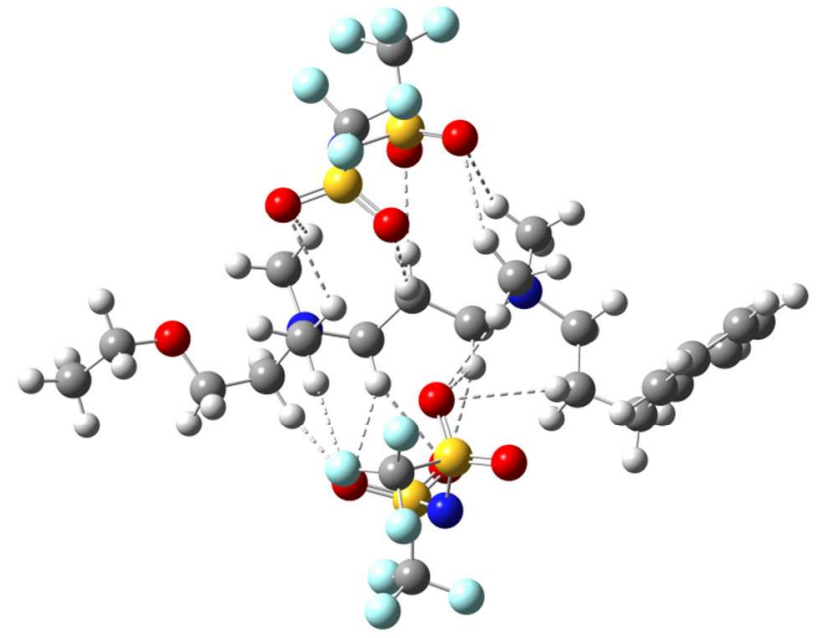

(e) 7.8

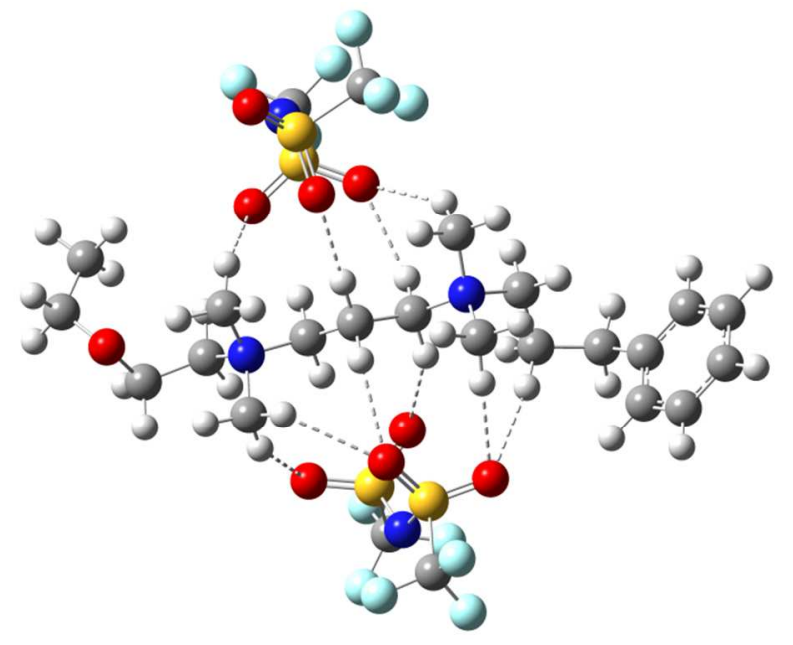

(g) 11.0

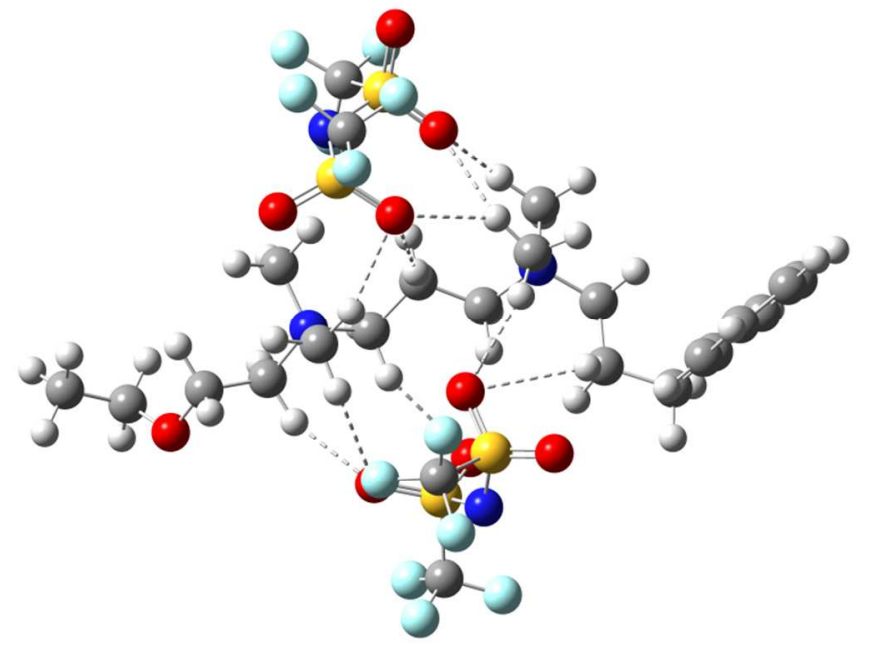

(f) 9.8

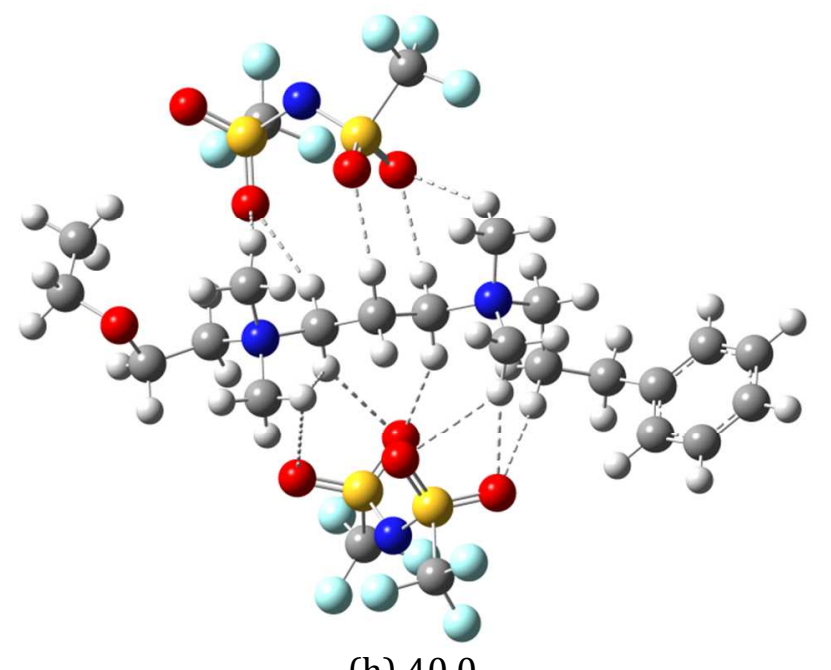

(h) 40.0

Figure S4 
Table S1(a): Hydrogen bonding distances (in $\AA$ ) (prime and without prime referring to two anions) in dication- $\left(\mathrm{Tf}_{2} \mathrm{~N}\right)_{2}$ complexes.

\begin{tabular}{|c|c|c|c|c|c|c|c|}
\hline \multicolumn{2}{|c|}{$[\mathrm{DRR} 1]\left(\mathrm{Tf}_{2} \mathrm{~N}\right)_{2}$} & \multicolumn{2}{|c|}{ [DRR2](Tf2N) ${ }_{2}$} & \multicolumn{2}{|c|}{$[\mathrm{DRR} 3]\left(\mathrm{Tf}_{2} \mathrm{~N}\right)_{2}$} & \multicolumn{2}{|c|}{$[\mathrm{DRR} 4]\left(\mathrm{Tf}_{2} \mathrm{~N}\right)_{2}$} \\
\hline \multicolumn{8}{|c|}{ Distances $(\AA)$} \\
\hline 01---H1 & 2.482 & 01---H3 & 2.412 & 04---H21 & 2.625 & 01---H3 & 2.409 \\
\hline 01---H7 & 2.312 & O3---H7 & 2.408 & O4---H7 & 2.435 & 01---H13 & 2.376 \\
\hline 01---H18 & 2.575 & 01---H5 & 2.471 & O4---H1 & 2.334 & 01---H5 & 2.505 \\
\hline O2---H20 & 2.483 & 01---H13 & 2.423 & O3---H3 & 2.418 & 03---H7 & 2.416 \\
\hline O2---H5 & 2.452 & 01---H1 & 2.646 & O3---H5 & 2.501 & O3---H18 & 2.580 \\
\hline O2---H1 & 2.630 & 01---H3 & 2.359 & 01---H19 & 2.307 & O3---H1 & 2.461 \\
\hline 04---H3 & 2.284 & 04---H1 & 2.469 & 01---H5 & 2.366 & 02---H17 & 2.479 \\
\hline O4---H13 & 2.371 & O4---H20 & 2.580 & 01---H14 & 2.292 & O4---H5 & 2.412 \\
\hline 01'---H15 & 2.462 & O2'---H4 & 2.353 & O4'---H4 & 2.267 & O4---H1 & 2.426 \\
\hline 01'---H6 & 2.255 & O2'---H16 & 2.531 & O4'---H10 & 2.436 & O2'---H4 & 2.339 \\
\hline 01'---H17 & 2.506 & O3'---H10 & 2.357 & O4'---H16 & 2.564 & O2'---H14 & 2.522 \\
\hline 01'---H2 & 2.595 & O3'---H2 & 2.283 & O2'---H11 & 2.329 & O1'---H11 & 2.563 \\
\hline O2'---H10 & 2.535 & O1'---H10 & 2.584 & O2'---H2 & 2.480 & O4'---H15 & 2.420 \\
\hline O2'---H12 & 2.541 & O4'---H6 & 2.466 & O1'---H18 & 2.388 & O4'---H6 & 2.473 \\
\hline O3'---H16 & 2.328 & O4'---H15 & 2.404 & 01'---H6 & 2.273 & O3'---H12 & 2.371 \\
\hline O3'---H15 & 2.653 & & & F79---H5 & 2.574 & O3'---H2 & 2.257 \\
\hline 013---H19 & 2.203 & 013---H12 & 2.268 & & & & \\
\hline 017---H9 & 2.240 & & & & & & \\
\hline
\end{tabular}


Table S1(b): Selected bond- distances (in $\AA$ ) and -angles (in ${ }^{\circ}$ ) of isolated dications and dication-(Tf $\left.{ }_{2}\right)_{2}$ complexes.

\begin{tabular}{|c|c|c|c|c|c|c|c|c|}
\hline & {$[\mathrm{DRR} 1]^{2+}$} & [DRR1] $\left(\mathrm{Tf}_{2} \mathrm{~N}\right)_{2}$ & {$[\mathrm{DRR} 2]^{2+}$} & [DRR2] $(\mathrm{Tf} 2 \mathrm{~N})_{2}$ & {$[\mathrm{DRR} 3]^{2+}$} & [DRR3] $\left(\mathrm{Tf}_{2} \mathrm{~N}\right)_{2}$ & {$[\text { DRR4] }]^{2+}$} & {$\left[\right.$ DRR4] $\left(\mathrm{Tf}_{2} \mathrm{~N}\right)_{2}$} \\
\hline \multicolumn{9}{|c|}{ Distances $(\AA)$} \\
\hline C1-H1 & 1.094 & 1.091 & 1.095 & 1.087 & 1.095 & 1.092 & 1.094 & 1.088 \\
\hline $\mathrm{C} 2-\mathrm{H} 3$ & 1.092 & 1.090 & 1.092 & 1.090 & 1.092 & 1.090 & 1.092 & 1.090 \\
\hline $\mathrm{C} 2-\mathrm{H} 4$ & 1.092 & 1.092 & 1.091 & 1.089 & 1.092 & 1.090 & 1.092 & 1.089 \\
\hline $\mathrm{C} 3-\mathrm{H} 5$ & 1.091 & 1.093 & 1.092 & 1.088 & 1.095 & 1.089 & 1.092 & 1.088 \\
\hline $\mathrm{C} 3-\mathrm{H} 6$ & 1.094 & 1.088 & 1.092 & 1.094 & 1.094 & 1.091 & 1.095 & 1.094 \\
\hline $\mathrm{C} 4-\mathrm{H} 8$ & 1.091 & 1.089 & 1.092 & 1.088 & 1.091 & 1.089 & 1.090 & 1.088 \\
\hline C4-H9 & 1.088 & 1.087 & 1.090 & 1.090 & 1.089 & 1.088 & 1.094 & 1.090 \\
\hline C5-H10 & 1.090 & 1.090 & 1.091 & 1.091 & 1.090 & 1.090 & 1.091 & 1.091 \\
\hline C5-H11 & 1.092 & 1.089 & 1.092 & 1.091 & 1.092 & 1.090 & 1.088 & 1.088 \\
\hline C5-H12 & 1.090 & 1.090 & 1.088 & 1.088 & 1.090 & 1.090 & 1.092 & 1.091 \\
\hline C6-H13 & 1.090 & 1.091 & 1.090 & 1.089 & 1.090 & 1.089 & 1.089 & 1.088 \\
\hline C6-H14 & 1.090 & 1.092 & 1.090 & 1.089 & 1.092 & 1.091 & 1.090 & 1.090 \\
\hline \multicolumn{9}{|c|}{ Angles (deg) } \\
\hline $\mathrm{a}(\mathrm{N} 2 \mathrm{C} 7 \mathrm{H} 16)$ & 109.2 & 107.5 & 108.9 & 109.4 & 109.3 & 108.4 & 109.7 & 110.0 \\
\hline $\mathrm{a}(\mathrm{N} 1 \mathrm{C} 4 \mathrm{H} 7)$ & 108.3 & 108.3 & 108.9 & 108.5 & 108.2 & 108.1 & 108.9 & 108.6 \\
\hline $\mathrm{a}(\mathrm{C} 8 \mathrm{C} 9 \mathrm{H} 20)$ & 112.8 & 112.4 & 118.0 & 117.8 & 111.0 & 109.9 & 111.7 & 111.2 \\
\hline $\mathrm{d}(\mathrm{H} 10 \mathrm{C} 5 \mathrm{~N} 1 \mathrm{C} 1)$ & 58.7 & -63.3 & -71.9 & 50.8 & 58.2 & 56.5 & -71.5 & -69.1 \\
\hline $\mathrm{d}(\mathrm{N} 1 \mathrm{C} 1 \mathrm{C} 2 \mathrm{C} 3)$ & 174.8 & 179.0 & 178.3 & 170.4 & 172.2 & -174.9 & 175.2 & 171.7 \\
\hline $\mathrm{d}(\mathrm{N} 2 \mathrm{C} 3 \mathrm{C} 2 \mathrm{C} 1)$ & 174.3 & 178.5 & -178.5 & -175.7 & 172.2 & -176.8 & 174.1 & 174.3 \\
\hline
\end{tabular}


Table S1(c): Selected bond- distances (in $\AA$ ) and -angles (in ${ }^{\circ}$ ) in the isolated $\left[\mathrm{Tf}_{2} \mathrm{~N}\right]^{-}$anion and dication- $\left(\mathrm{Tf}_{2} \mathrm{~N}\right)_{2}$ complexes.

\begin{tabular}{|c|c|c|c|c|c|c|c|c|c|}
\hline & \multirow[t]{2}{*}[\mathrm{Tf}_{2}\mathrm{N}]{$^{-}$} & \multicolumn{2}{|c|}{$[\mathrm{DRR} 1]\left(\mathrm{Tf}_{2} \mathrm{~N}\right)_{2}$} & \multicolumn{2}{|c|}{$[\mathrm{DRR} 2](\mathrm{Tf} 2 \mathrm{~N})_{2}$} & \multicolumn{2}{|c|}{$[\mathrm{DRR} 3]\left(\mathrm{Tf}_{2} \mathrm{~N}\right)_{2}$} & \multicolumn{2}{|c|}{ [DRR4] $\left(\mathrm{Tf}_{2} \mathrm{~N}\right)_{2}$} \\
\hline & & (a) & (b) & (a) & (b) & (a) & (b) & (a) & (b) \\
\hline \multicolumn{10}{|c|}{ Distances $(\AA ̊)$} \\
\hline S1-01 & 1.476 & 1.480 & 1.483 & 1.492 & 1.475 & 1.476 & 1.486 & 1.492 & 1.476 \\
\hline $\mathrm{S} 2-03$ & 1.476 & 1.472 & 1.480 & 1.475 & 1.492 & 1.485 & 1.487 & 1.487 & 1.483 \\
\hline $\mathrm{S} 1-02$ & 1.477 & 1.479 & 1.480 & 1.481 & 1.485 & 1.488 & 1.466 & 1.474 & 1.486 \\
\hline $\mathrm{S} 2-04$ & 1.477 & 1.485 & 1.483 & 1.485 & 1.471 & 1.482 & 1.487 & 1.484 & 1.481 \\
\hline S1-N & 1.624 & 1.621 & 1.612 & 1.612 & 1.611 & 1.609 & 1.609 & 1.609 & 1.612 \\
\hline S2-N & 1.624 & 1.629 & 1.624 & 1.628 & 1.623 & 1.631 & 1.616 & 1.626 & 1.629 \\
\hline $\mathrm{C}-\mathrm{F}$ & $1.334-1.342$ & $1.329-1.337$ & $1.330-1.332$ & $1.328-1.336$ & $1.330-1.332$ & $1.325-1.345$ & $1.329-1.333$ & $1.330-1.332$ & $1.328-1.335$ \\
\hline \multicolumn{10}{|c|}{ Angles (deg) } \\
\hline $\mathrm{a}(01 \mathrm{~S} 102)$ & 119.2 & 118.1 & 117.8 & 118.0 & 116.1 & 117.5 & 119.5 & 117.7 & 118.1 \\
\hline $\mathrm{a}(03 \mathrm{O} 204)$ & 119.2 & 119.1 & 117.6 & 118.3 & 118.4 & 117.7 & 115.5 & 116.3 & 118.1 \\
\hline $\mathrm{a}(\mathrm{S} 1 \mathrm{NS} 2)$ & 121.3 & 116.5 & 121.7 & 116.7 & 121.9 & 122.5 & 119.2 & 116.5 & 121.7 \\
\hline $\mathrm{a}(01 \mathrm{~S} 1 \mathrm{~N})$ & 117.3 & 115.6 & 115.8 & 107.6 & 116.0 & 114.9 & 115.4 & 113.9 & 117.2 \\
\hline$a(03 S 2 N)$ & 117.3 & 116.9 & 116.9 & 109.6 & 113.6 & 116.8 & 117.2 & 115.7 & 115.6 \\
\hline $\mathrm{a}(\mathrm{O} 2 \mathrm{~S} 1 \mathrm{~N})$ & 108.0 & 105.9 & 107.4 & 115.8 & 113.9 & 109.0 & 108.9 & 111.8 & 109.8 \\
\hline $\mathrm{a}(04 \mathrm{~S} 2 \mathrm{~N})$ & 108.0 & 108.1 & 109.9 & 117.1 & 111.7 & 111.6 & 113.8 & 113.8 & 107.9 \\
\hline$d(01 S 1 N S 2)$ & -22.1 & -34.6 & -20.8 & -152.9 & -64.7 & -13.8 & -28.8 & -2.5 & -5.6 \\
\hline $\mathrm{d}(03 \mathrm{~S} 2 \mathrm{NS} 1)$ & -22.1 & -3.0 & -16.3 & 113.8 & -4.5 & -42.7 & 37.6 & -65.4 & -18.3 \\
\hline
\end{tabular}



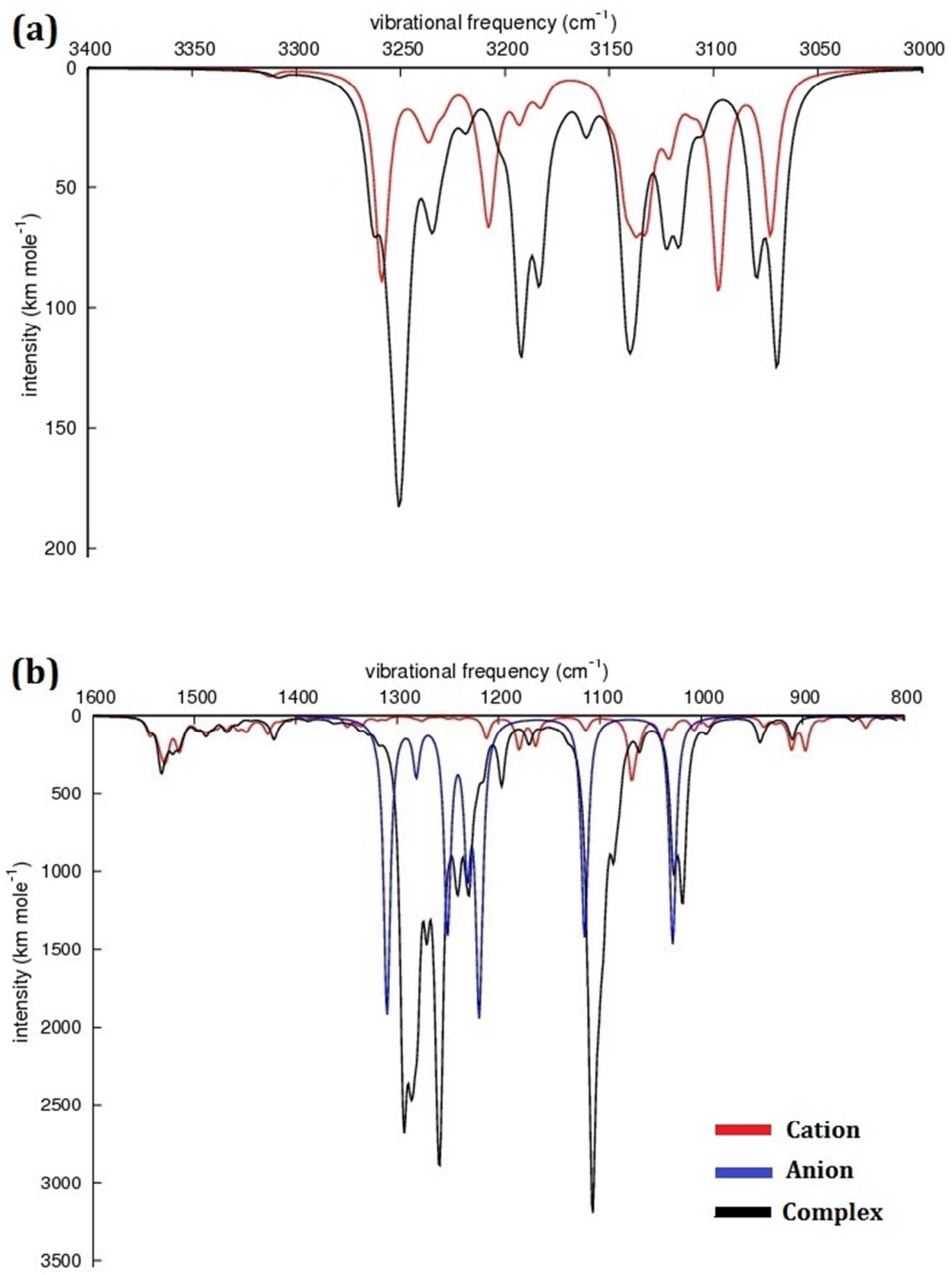

Figure S5 

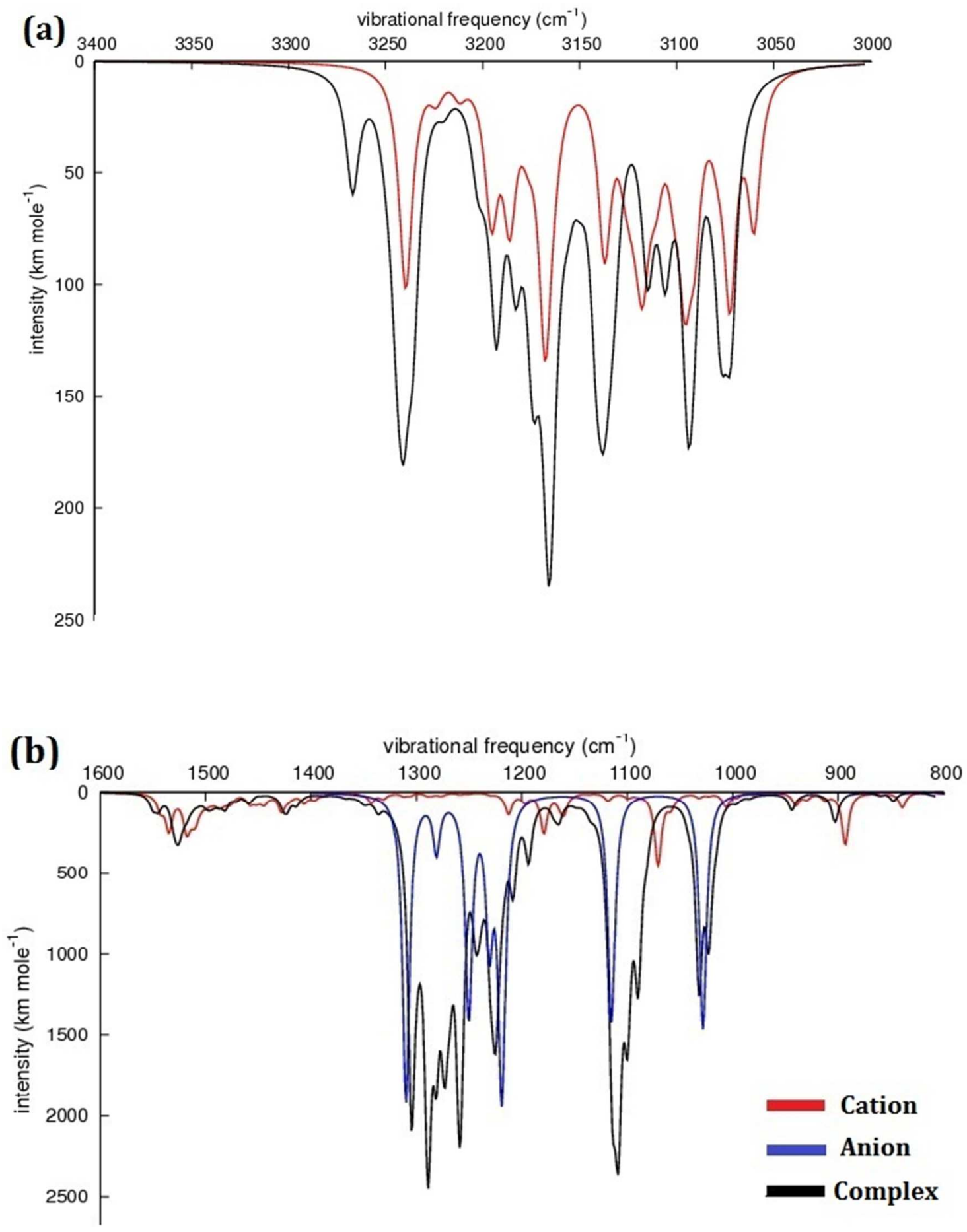

Figure S6 

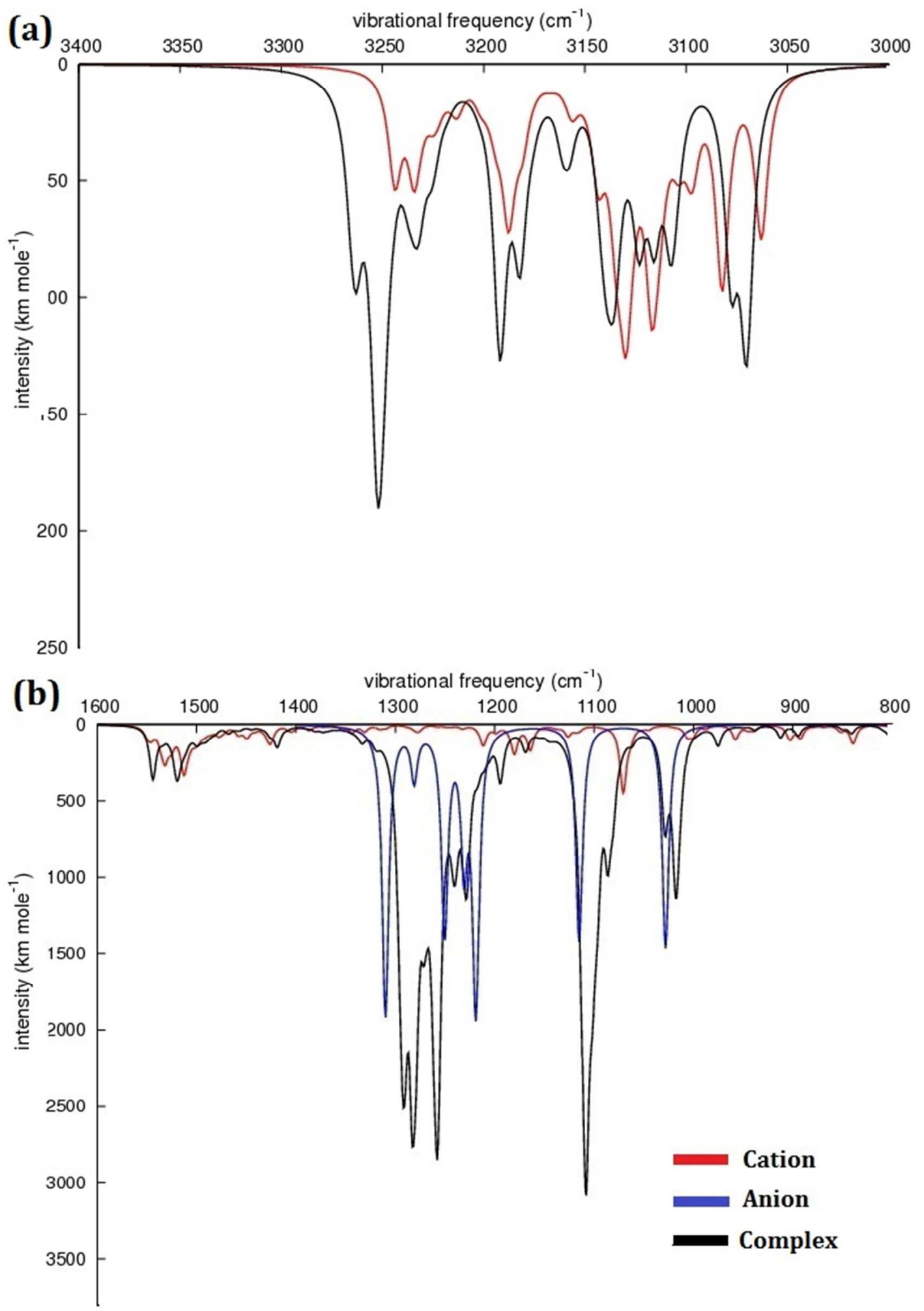

Figure S7 
Table S2: Selected charges (in a.u.) driven from the NBO analysis in isolated $\mathrm{Tf}_{2} \mathrm{~N}^{-}$anion and their dication- $\left(\mathrm{Tf}_{2} \mathrm{~N}\right)_{2}$ complexes.

\begin{tabular}{|c|c|c|c|c|c|c|c|c|c|}
\hline \multirow[t]{2}{*}{ Atoms } & \multirow[t]{2}{*}{$\mathrm{Tf}_{2} \mathrm{~N}^{-}$} & \multicolumn{2}{|c|}{ [DRR1] $\left(\mathrm{Tf}_{2} \mathrm{~N}\right)_{2}$} & \multicolumn{2}{|c|}{ [DRR2](Tf2N) ${ }_{2}$} & \multicolumn{2}{|c|}{ [DRR3] $\left(\mathrm{Tf}_{2} \mathrm{~N}\right)_{2}$} & \multicolumn{2}{|c|}{$[\mathrm{DRR} 4]\left(\mathrm{Tf}_{2} \mathrm{~N}\right)_{2}$} \\
\hline & & (a) & (b) & (a) & (b) & (a) & (b) & (a) & (b) \\
\hline $\mathrm{S} 1$ & 2.232 & 2.233 & 2.238 & 2.243 & 2.220 & 2.256 & 2.236 & 2.244 & 2.219 \\
\hline $\mathrm{S} 2$ & 2.232 & 2.225 & 2.225 & 2.212 & 2.239 & 2.221 & 2.215 & 2.211 & 2.239 \\
\hline 01 & -0.950 & -0.961 & -0.979 & -1.017 & -0.937 & -0.922 & -0.989 & -1.013 & -0.939 \\
\hline 02 & -0.962 & -0.961 & -0.961 & -0.938 & -0.973 & -0.999 & -0.967 & -0.948 & -0.972 \\
\hline 03 & -0.950 & -0.925 & -0.951 & -0.979 & -0.968 & -0.984 & -0.940 & -0.977 & -0.977 \\
\hline 04 & -0.962 & -0.974 & -0.968 & -0.968 & -0.968 & -0.979 & -0.979 & -0.963 & -0.966 \\
\hline $\mathrm{N}$ & -1.231 & -1.268 & -1.268 & -1.214 & -1.256 & -1.217 & -1.241 & -1.213 & -1.254 \\
\hline $\mathrm{C} 1$ & 0.935 & 0.960 & 0.959 & 0.951 & 0.962 & 0.942 & 0.960 & 2.244 & 2.219 \\
\hline $\mathrm{C} 2$ & 0.935 & 0.954 & 0.962 & 0.976 & 0.957 & 0.962 & 0.967 & 2.210 & 2.239 \\
\hline
\end{tabular}




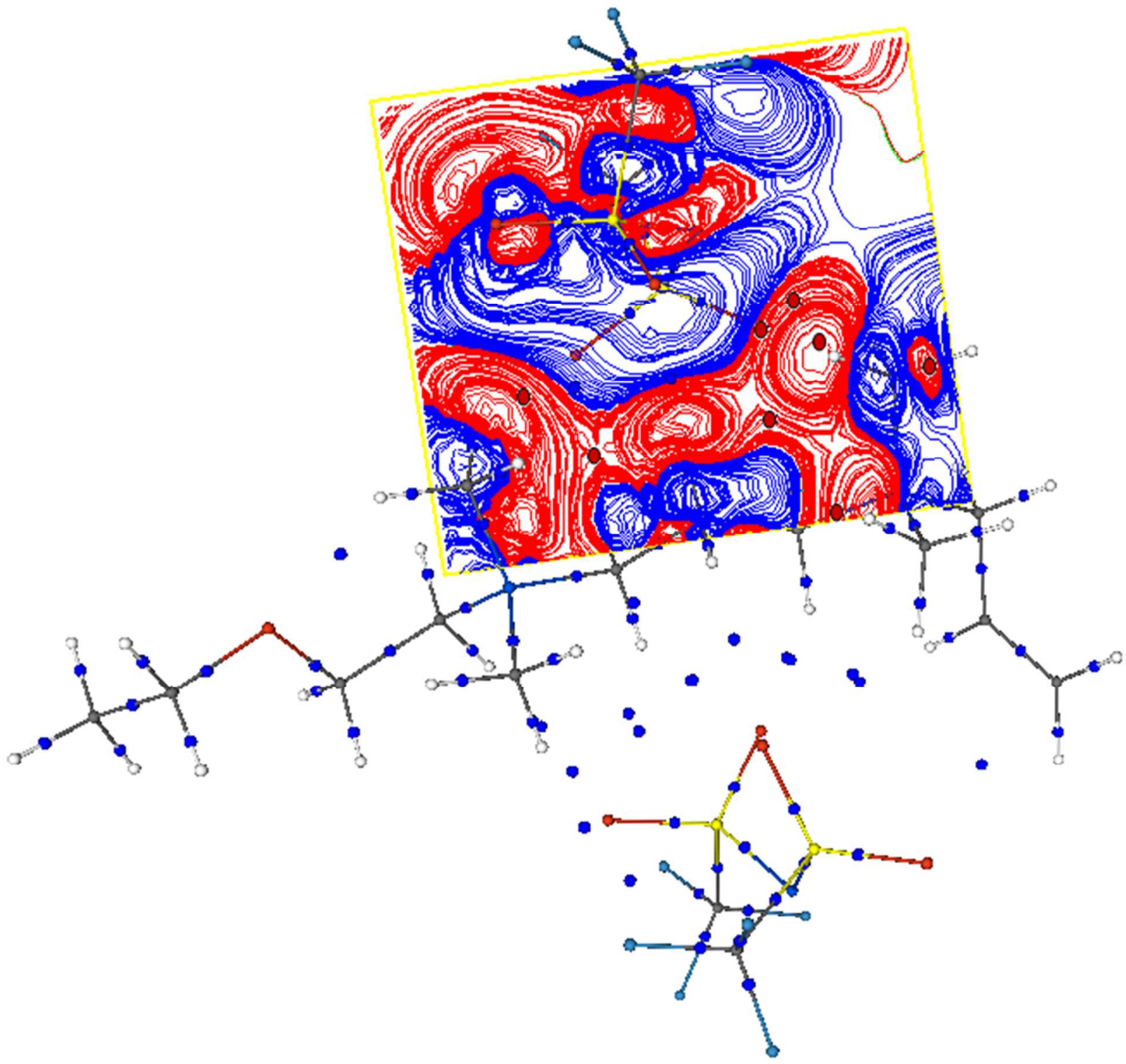

Figure S8 


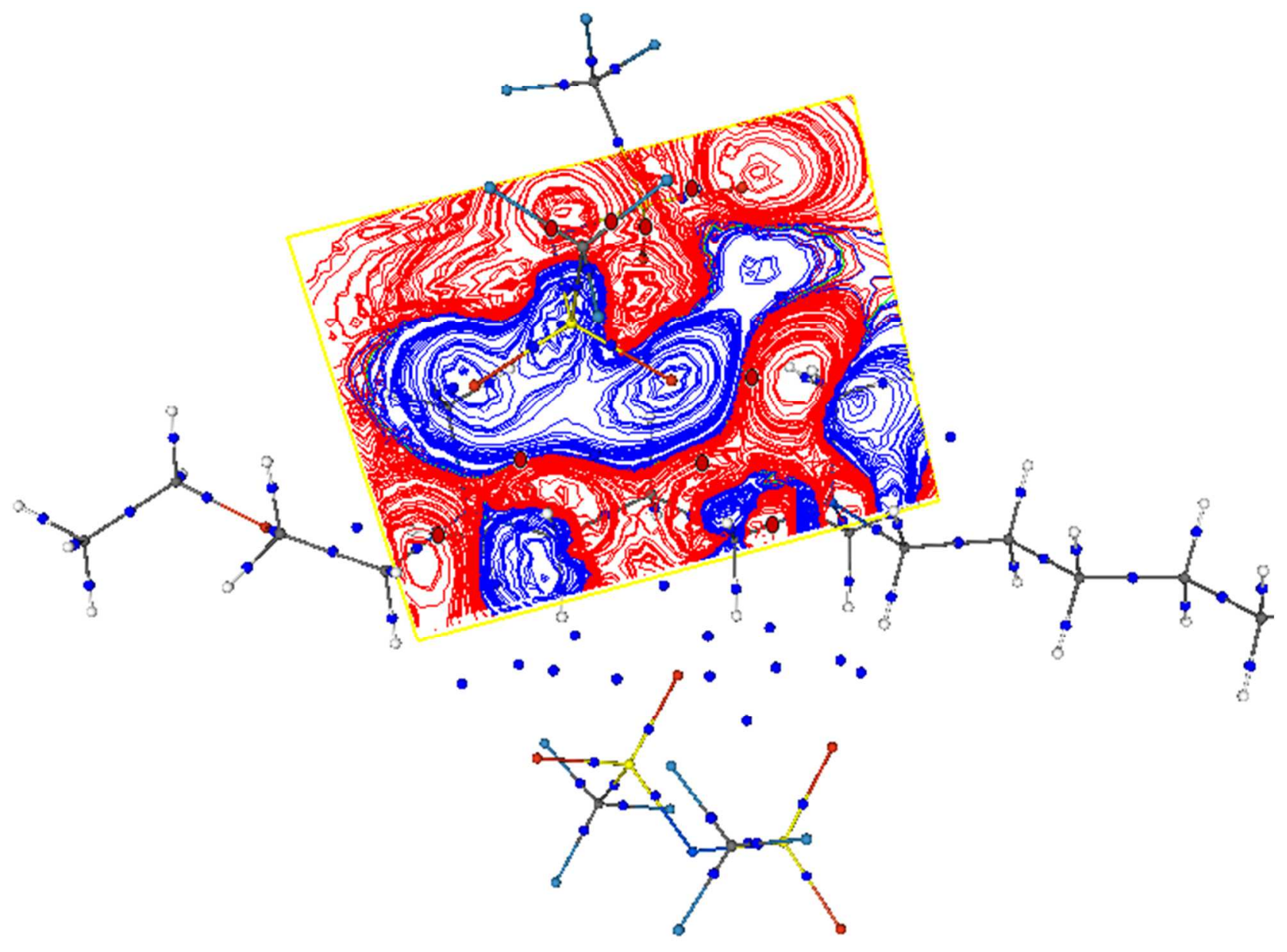

Figure S9 


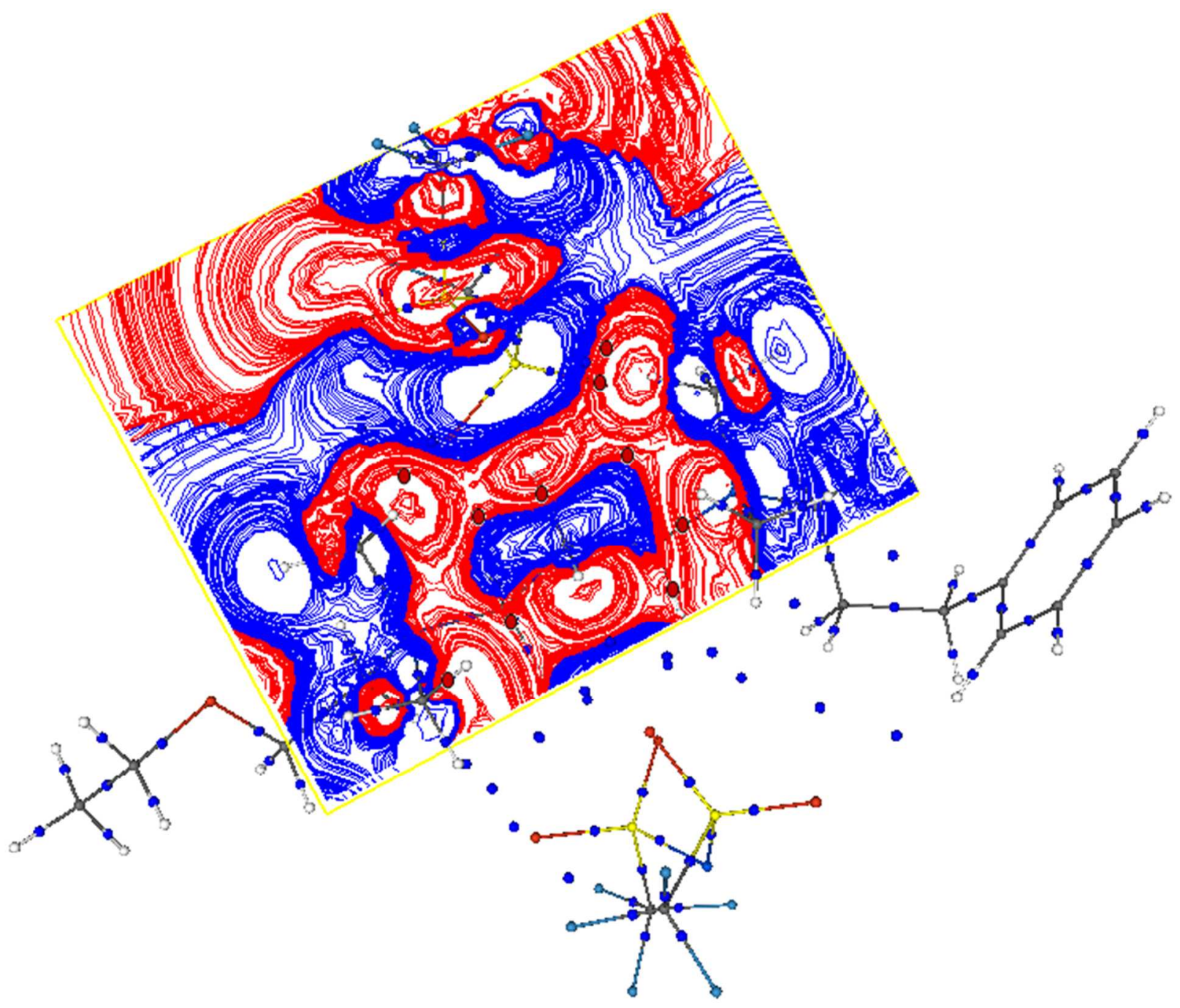

Figure S10 

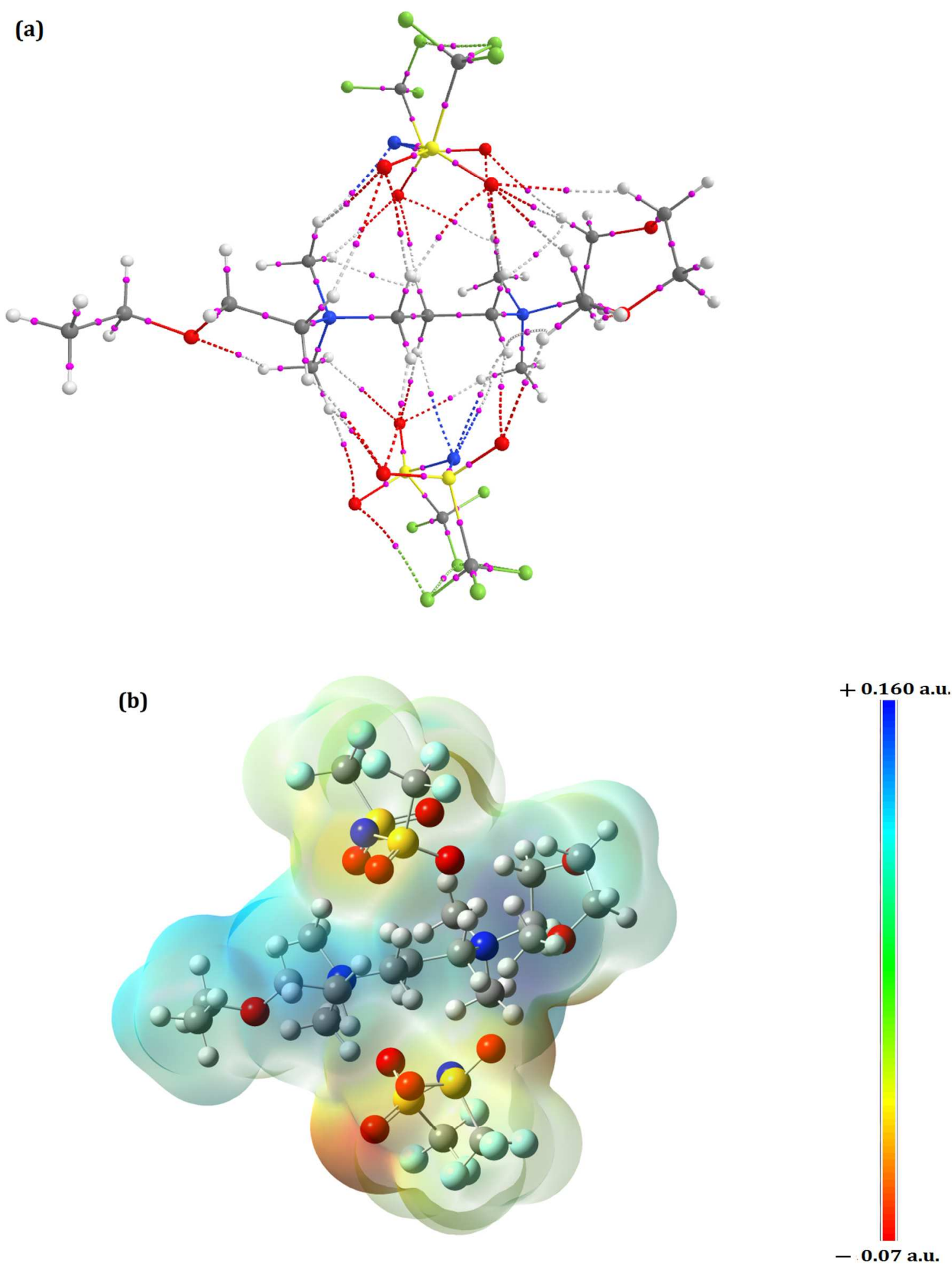

Figure S11 


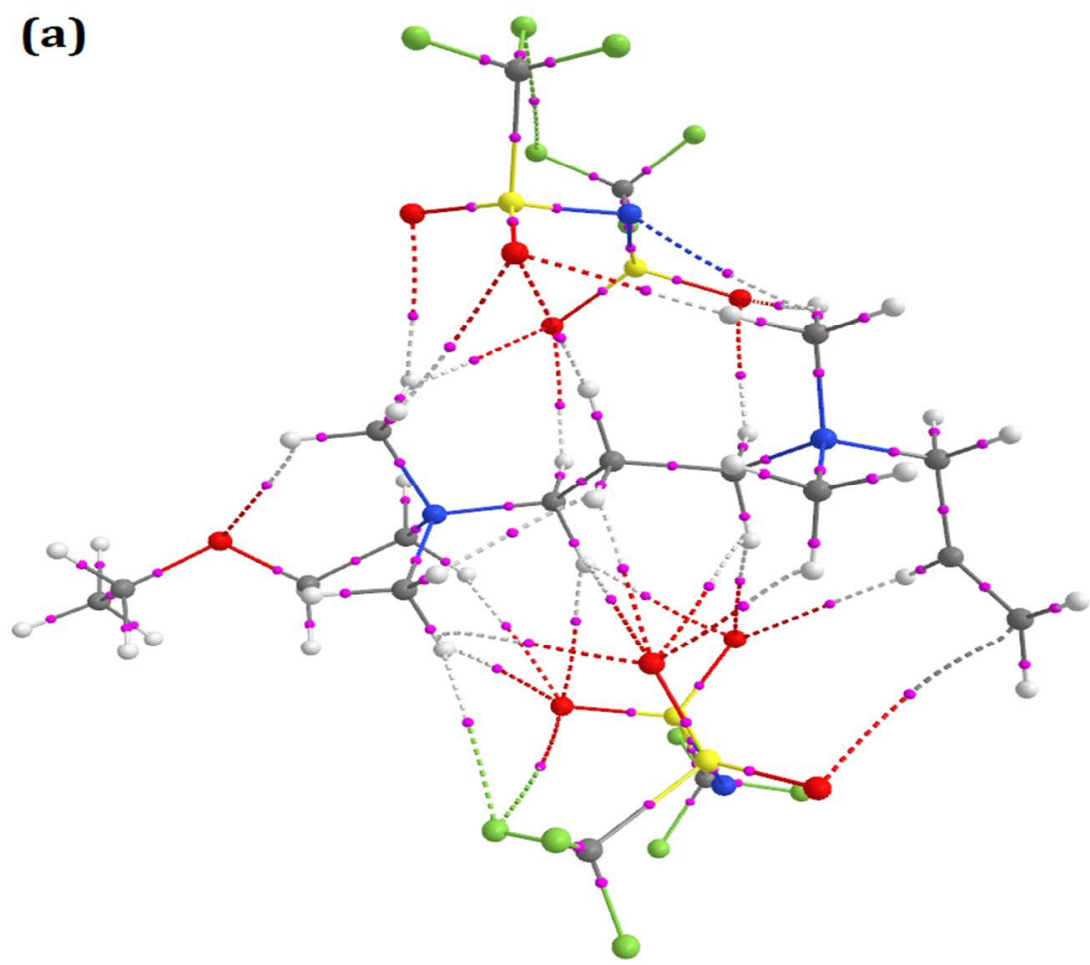

(b)
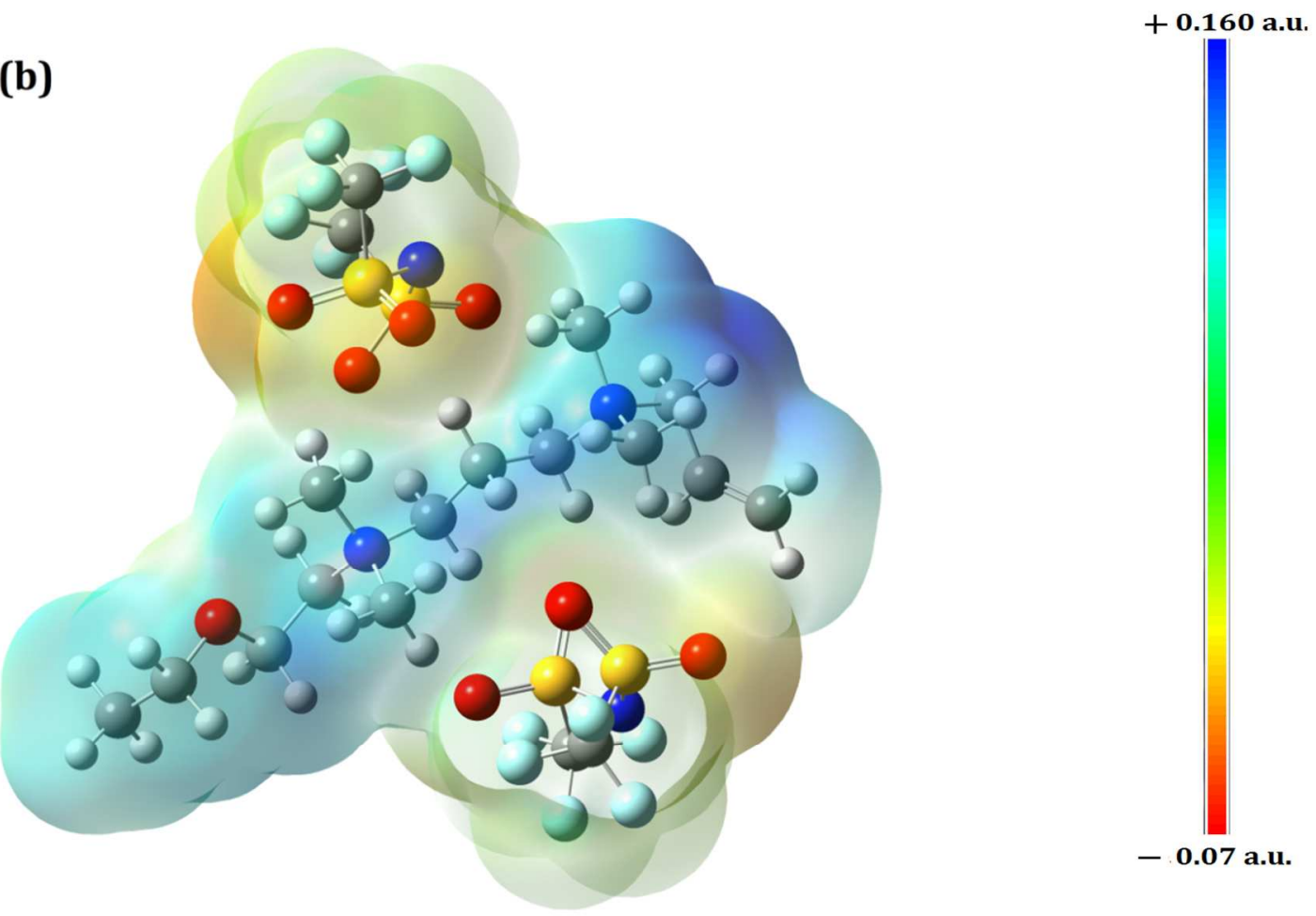

Figure S12 

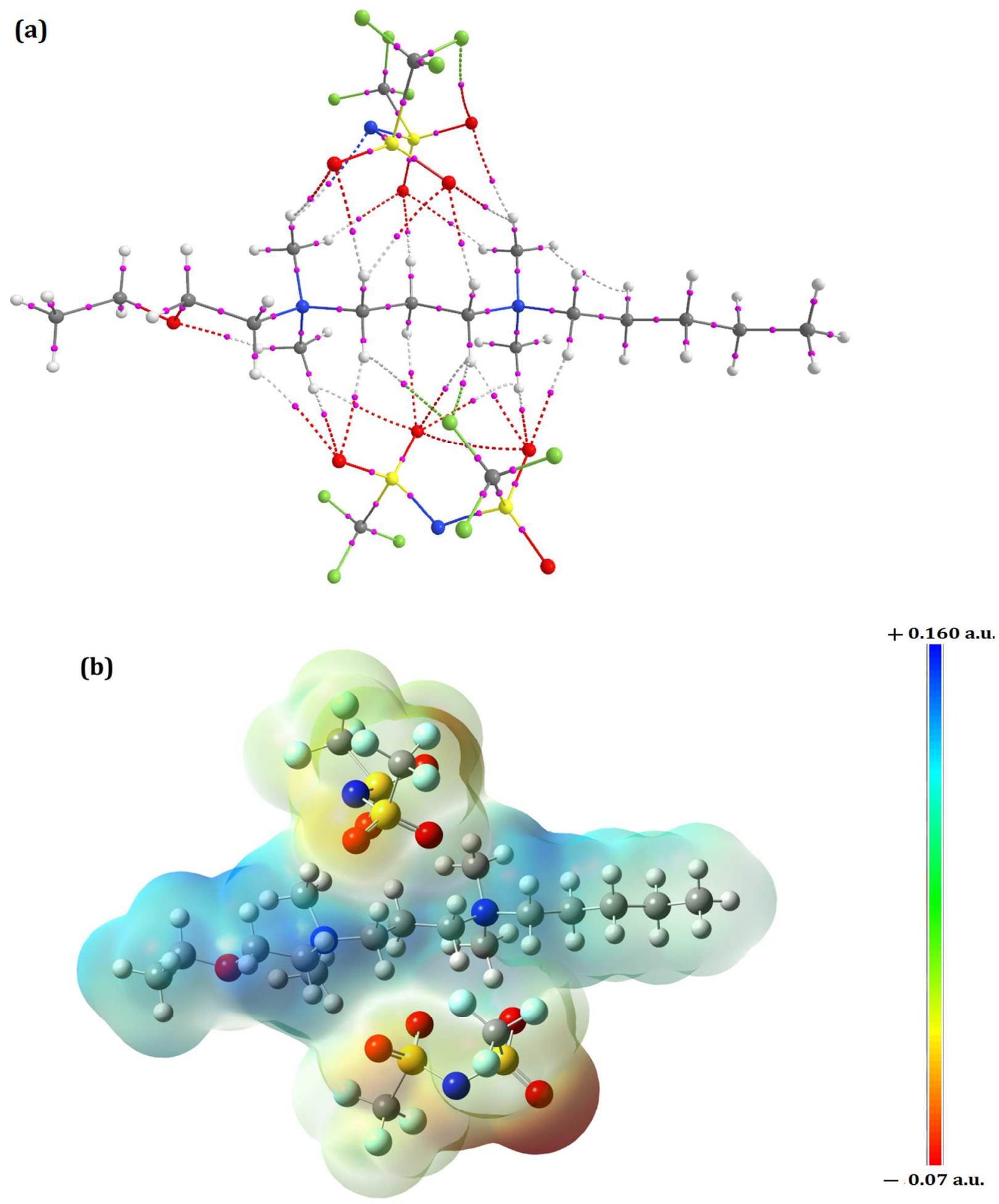

Figure S13 


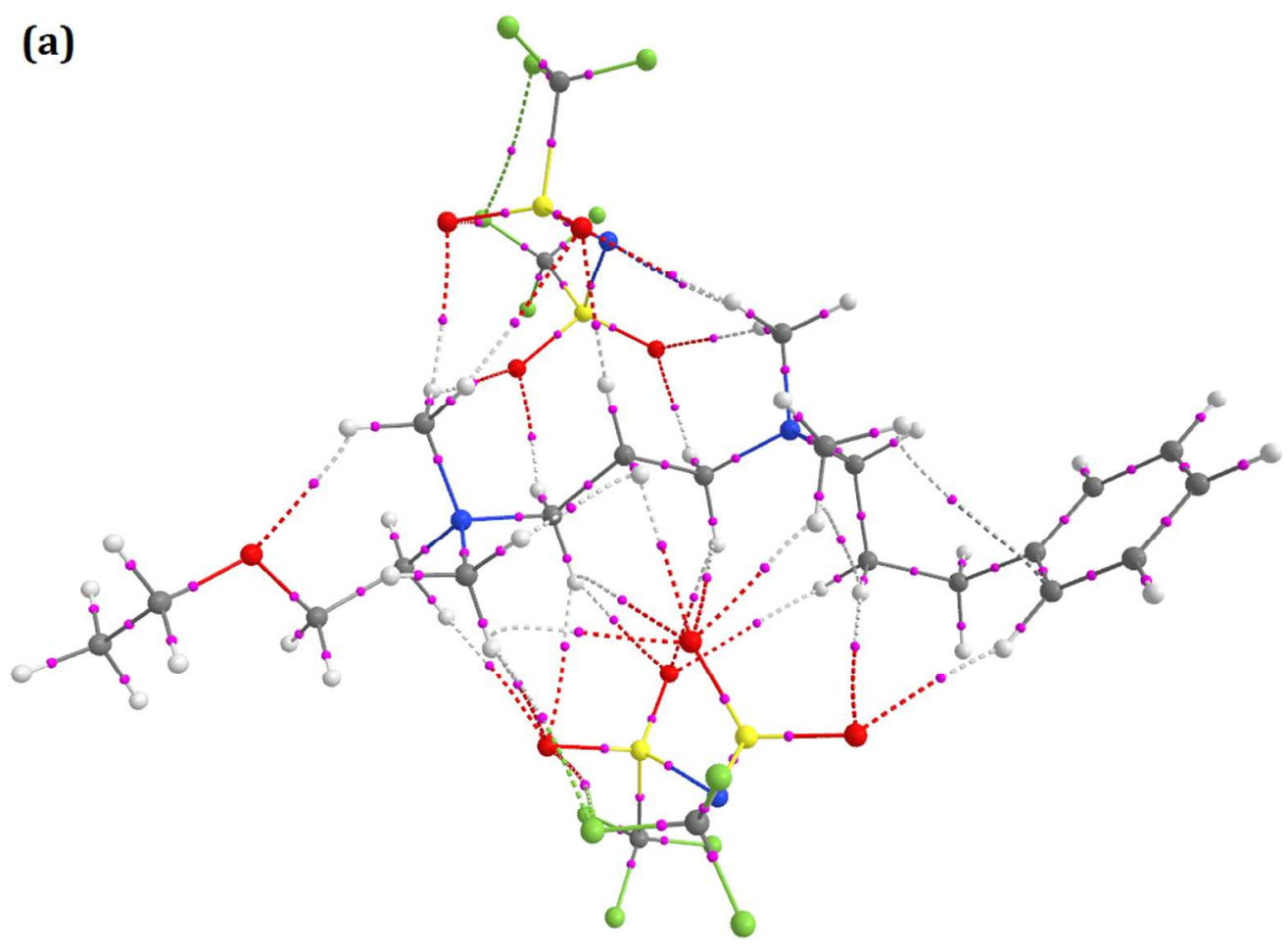

(b)
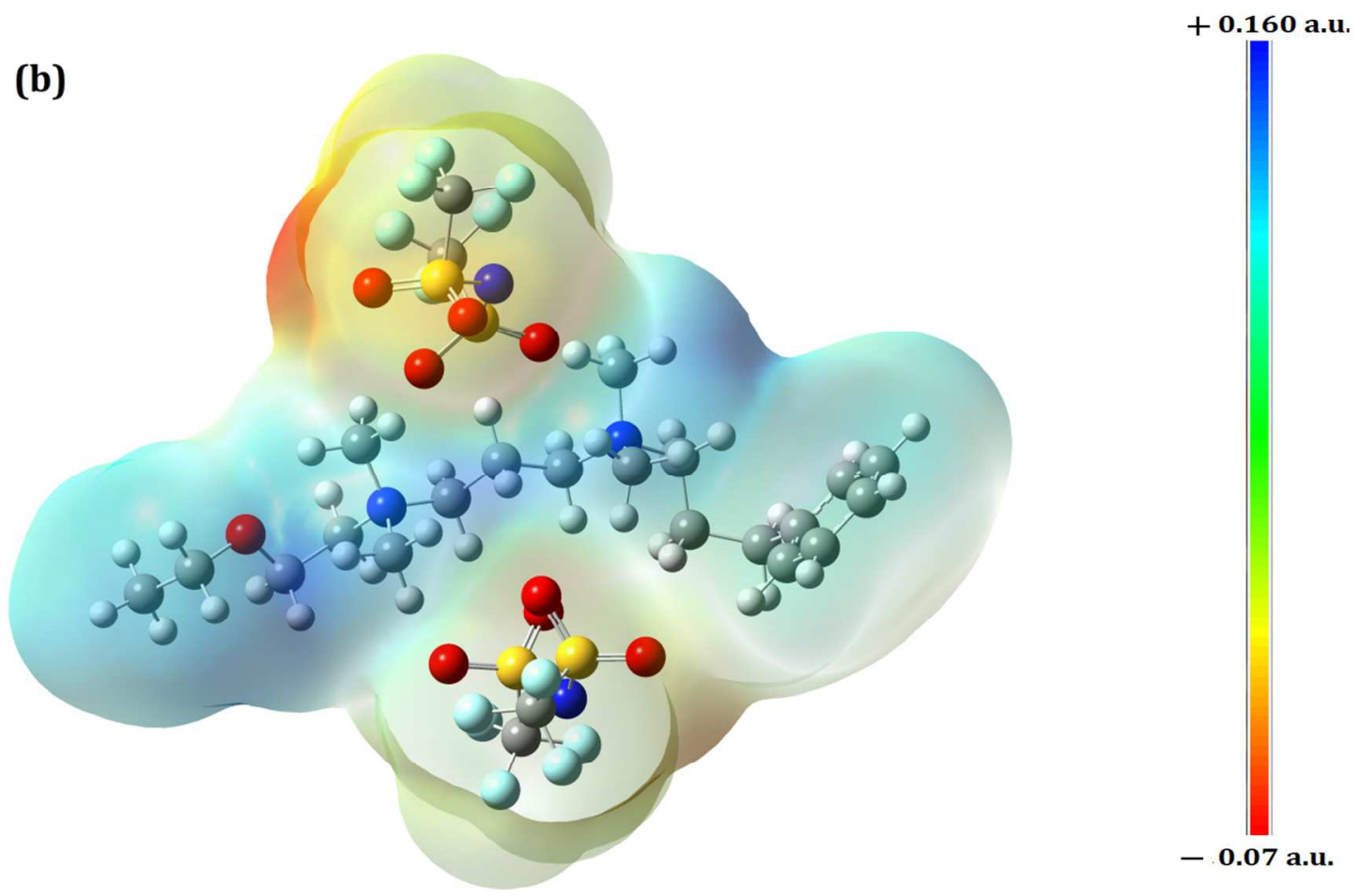

Figure S14 


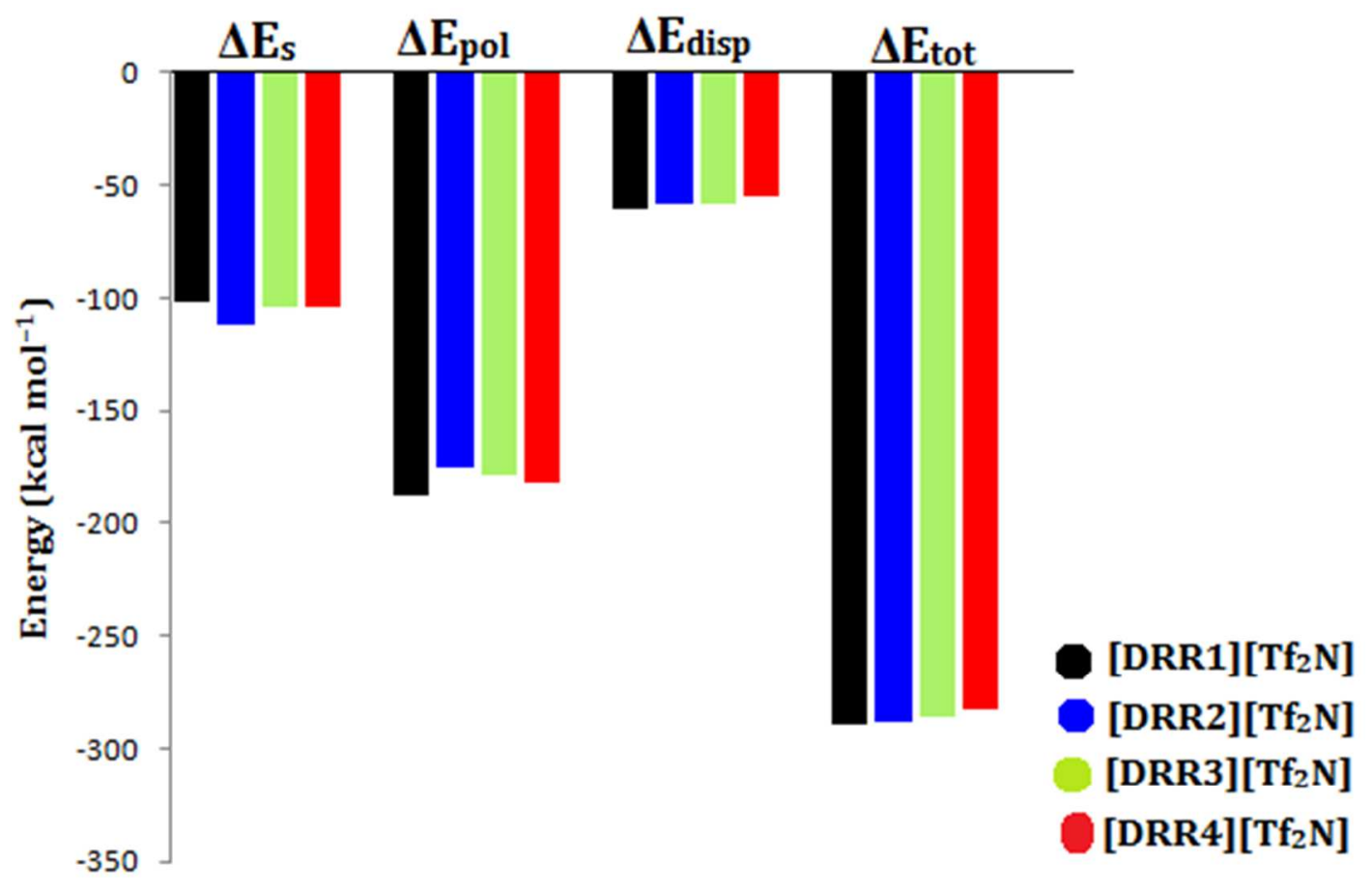

Figure S15 


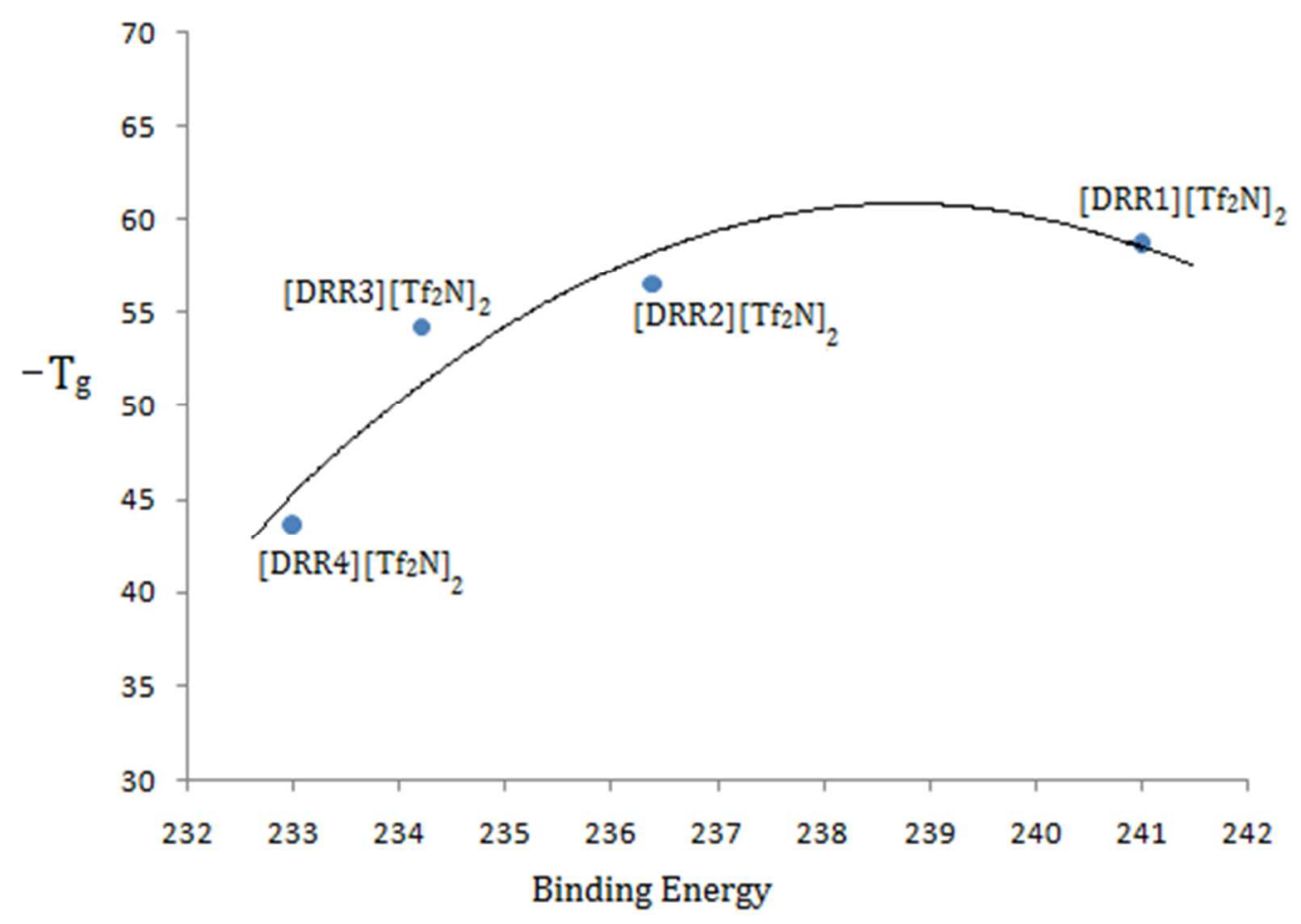

Figure S16

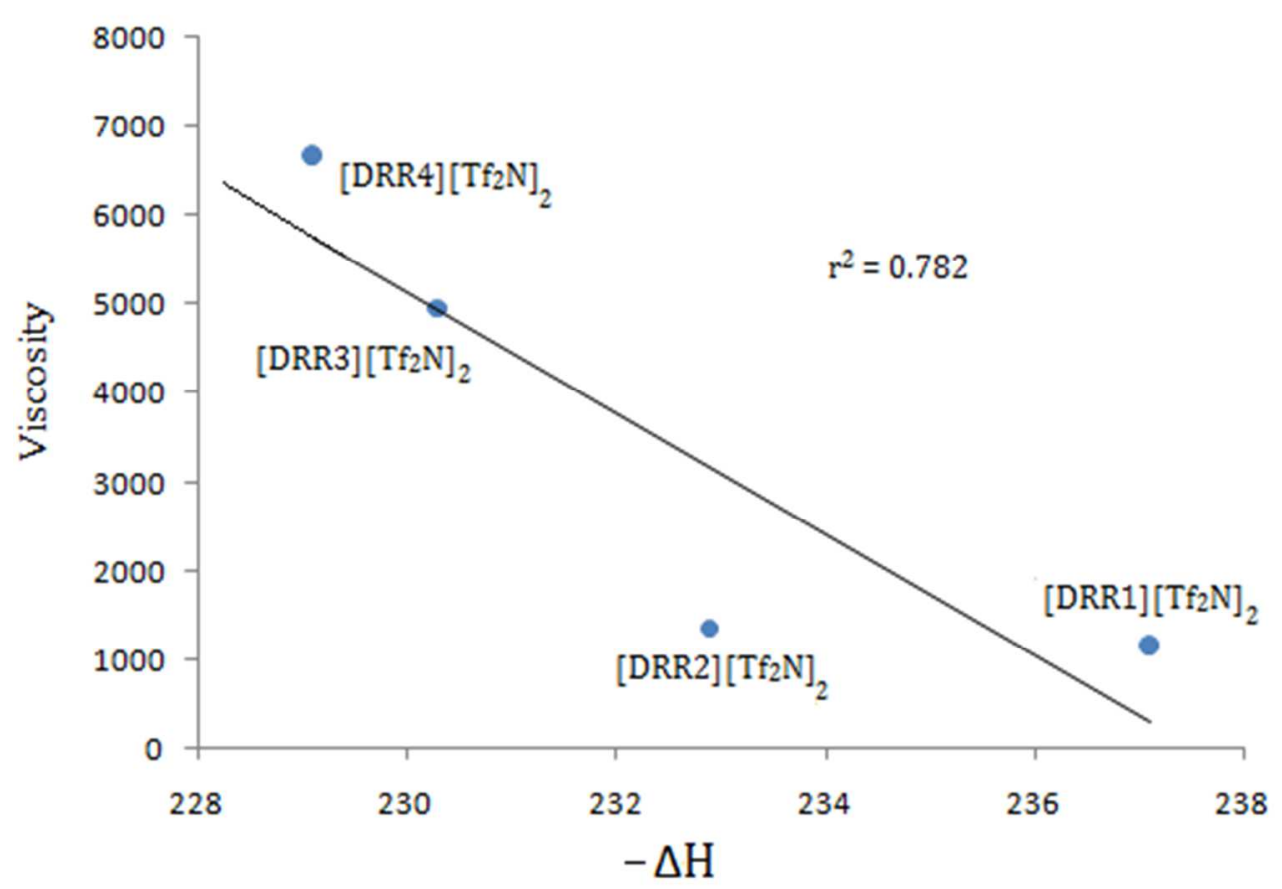

Figure S17 